\title{
DISTINGUISHED EXCHANGEABLE COALESCENTS AND GENERALIZED FLEMING-VIOT PROCESSES WITH IMMIGRATION
}

\author{
CLÉMENT FOUCART, ${ }^{*}$ Université Pierre et Marie Curie
}

\begin{abstract}
Coalescents with multiple collisions (also called $\Lambda$-coalescents or simple exchangeable coalescents) are used as models of genealogies. We study a new class of Markovian coalescent processes connected to a population model with immigration. Consider an infinite population with immigration labelled at each generation by $\mathbb{N}:=\{1,2, \ldots\}$. Some ancestral lineages cannot be followed backwards after some time because their ancestor is outside the population. The individuals with an immigrant ancestor constitute a distinguished family and we define exchangeable distinguished coalescent processes as a model for genealogy with immigration, focusing on simple distinguished coalescents, i.e. such that when a coagulation occurs all the blocks involved merge as a single block. These processes are characterized by two finite measures on $[0,1]$ denoted by $M=\left(\Lambda_{0}, \Lambda_{1}\right)$. We call them $M$-coalescents. We show by martingale arguments that the condition of coming down from infinity for the $M$-coalescent coincides with that obtained by Schweinsberg for the $\Lambda$-coalescent. In the same vein as Bertoin and Le Gall, $M$-coalescents are associated with some stochastic flows. The superprocess embedded can be viewed as a generalized Fleming-Viot process with immigration. The measures $\Lambda_{0}$ and $\Lambda_{1}$ respectively specify the reproduction and the immigration. The coming down from infinity of the $M$-coalescent will be interpreted as the initial types extinction: after a certain time all individuals are immigrant children.
\end{abstract}

Keywords: Exchangeable partition; coalescent theory; genealogy for a population with immigration; stochastic flow; coming down from infinity

2010 Mathematics Subject Classification: Primary 60J25; 60G09

Secondary 92D25

\section{Introduction}

Pitman [22] and Sagitov [24] defined in 1999 the class of $\Lambda$-coalescent processes, sometimes also called simple exchangeable coalescents. These coalescent processes appear as models for the genealogy of certain haploid populations with fixed size. The general motivation of this work is to define a new class of coalescent processes that may be used to describe the genealogy of a population with immigration. Heuristically, let us imagine an infinite haploid population with immigration described at each generation by $\mathbb{N}:=\{1,2, \ldots\}$. This means that each individual has at most one parent in the population at the previous generation; indeed, immigration implies that some individuals may have parents outside this population (they are children of immigrants). Sampling $n$ individuals in the population at some fixed generation, we group together the individuals with the same parent at the preceding generation. The individuals

Received 3 June 2010; revision received 3 September 2010.

* Postal address: Laboratoire de Probabilités et Modèles Aléatoires, Université Pierre et Marie Curie, 4 Place Jussieu, 75252 Paris Cedex 05, France. Email address: clement.foucart@etu.upmc.fr 
with an immigrant parent constitute a special family. We get a partition of $\mathbb{N}$ where each block is a family.

To give to the population a full genealogy, we may imagine a generic external ancestor, say 0 , to distinguish the immigrants family. This way, all families will have an ancestor at the preceding generation. Following the ancestral lineage of an individual backwards in time, it may coalesce with some others in $\mathbb{N}$ or reach 0 . In the latter case the lineage is absorbed at 0 . We call 0 the immigrant ancestor, and we will therefore work with partitions of $\mathbb{N} \cup\{0\}=\mathbb{Z}_{+}$. We view the block containing 0 as distinguished and then we speak of distinguished partitions. As usual, a partition is identified with the sequence of its blocks in the increasing order of their smallest element. The distinguished block is thus the first.

For a population with no immigration, Kingman introduced exchangeable random partitions of $\mathbb{N}$. A random partition is exchangeable if and only if its law is invariant under the action of permutations of $\mathbb{N}$. The distinguished partitions appearing in our setting are not exchangeable on $\mathbb{Z}_{+}$; however, their laws are invariant under the action of permutations $\sigma$ of $\mathbb{Z}_{+}$such that $\sigma(0)=0$. These partitions are called exchangeable distinguished partitions. We will present an extension of Kingman's theorem that determines their structure via a paint-box construction.

This allows us to define, following the approach in Bertoin's book [4, p. 177], a new class of coalescent processes, which we call exchangeable distinguished coalescents. An exchangeable distinguished coalescent is characterized in law by a measure $\mu^{0}$ on the space of partitions of $\mathbb{Z}_{+}$, called the distinguished coagulation measure. The extension of Kingman's theorem enables us to characterize this measure, and when $\mu^{0}$ is carried on the subset of simple distinguished partitions (which have only one nontrivial block), we get a representation involving two finite measures on $[0,1]: M=\left(\Lambda_{0}, \Lambda_{1}\right)$. We call this subclass of distinguished coalescents $M$-coalescents. The restriction of an $M$-coalescent to each finite subset containing 0 is a Markovian coalescent chain with the following transition rates. When the partition restricted to $\mathbb{N}$ has $b$ blocks, two kinds of jumps are allowed: for $b \geq k \geq 2$, each $k$-tuple of blocks not containing 0 can merge to form a single block at rate $\int_{0}^{1} x^{k-2}(1-x)^{b-k} \Lambda_{1}(\mathrm{~d} x)$, and, for $b \geq k \geq 1$, each $k$-tuple of blocks not containing 0 can merge with the one containing 0 at rate $\int_{0}^{1} x^{k-1}(1-x)^{b-k} \Lambda_{0}(\mathrm{~d} x)$.

Next, we study a classical phenomenon for coalescent processes: a coalescent process starting from infinitely many blocks is said to come down from infinity if its number of blocks instantaneously becomes finite. An interesting result is that the condition for $M$ coalescents to come down does not depend on $\Lambda_{0}$ and is the same for the $\Lambda_{1}$-coalescent found by Schweinsberg [25].

In the last section we define some stochastic flows connected with $M$-coalescents. The model of continuous population embedded in the flow can be viewed as a generalized FlemingViot process with immigration. As in [5], the stochastic flows involved allow us to define simultaneously a population model forwards in time and its genealogical process backwards in time. A duality between $M$-generalized Fleming-Viot processes with immigration and $M$-coalescents will be studied.

In a forthcoming paper, we will give a different approach to construct the generalized Fleming-Viot processes with immigration by introducing some stochastic flows of partitions. Our method will draw both on the works of Donnelly and Kurtz [13] and Bertoin and Le Gall [5]. Some ideas of Birkner et al. [8] may be applied to establish a link between certain branching processes with immigration and $M$-generalized Fleming-Viot processes with immigration, defined in [16]. 


\subsection{Outline}

The paper is organized as follows. In Section 2 we recall some basic facts on random partitions, and we give some fundamental properties of exchangeable distinguished partitions (the existence of asymptotic frequencies and paint-box representation). In Section 3 we define exchangeable distinguished coalescents. We establish a characterization of their laws by an exchangeable measure $\mu^{0}$ on the space of the distinguished partitions. The structure of $\mu^{0}$ is entirely described which enables us to study the dust. The main reference is Bertoin's book [4, Chapters 2,4]. The construction of exchangeable distinguished coalescents is very close to that for exchangeable coalescents of [4]. In Section 4 we focus on $M$-coalescents and study the coming down from infinity. In particular, our approach provides a new proof of Schweinsberg's result (see [25]) about the necessary and sufficient conditions to come down from infinity for $\Lambda$-coalescents based on martingale arguments. In Section 5 we introduce certain stochastic flows encoding $M$-coalescents. As in [4] and [5], these flows allow us to define a population model with immigration called $M$-generalized Fleming-Viot process with immigration.

\section{Distinguished partitions}

We begin with some general notation and properties which we will use constantly in the following sections.

For every integer $n \geq 1$, we denote by $[n]$ the set $\{1, \ldots, n\}$ and by $\mathcal{P}_{n}$ the set of its partitions. The set of partitions of $\mathbb{N}$ is denoted by $\mathcal{P}_{\infty}$. Let $\pi \in \mathcal{P}_{\infty}$, where we identify the set $\pi$ with the sequence $\left(\pi_{1}, \pi_{2}, \ldots\right)$ of the blocks of $\pi$ enumerated in increasing order of their least element: for every $i \leq j, \min \pi_{i} \leq \min \pi_{j}$. The number of blocks of $\pi$ is denoted $\# \pi$. For all $\pi \in \mathcal{P}_{\infty}$ and $n \in \mathbb{N}, \pi_{\mid[n]} \in \mathcal{P}_{n}$ is by definition the restriction of $\pi$ to [n]. We denote by $\mathcal{P}_{\mathrm{m}}$ the set of mass partitions, meaning the decreasing sequences with sum less than or equal to 1 :

$$
\mathcal{P}_{\mathrm{m}}:=\left\{s=\left(s_{1}, s_{2}, \ldots\right) ; \sum_{i \geq 1} s_{i} \leq 1, s_{1} \geq s_{2} \geq \cdots \geq 0\right\} .
$$

Given a partition $\pi=\left(B_{1}, B_{2}, \ldots\right)$ and a block $B$ of that partition, we say that $B$ has an asymptotic frequency, denoted by $|B|$, if the following limit exists:

$$
|B|:=\lim _{n \rightarrow \infty} \frac{\#(B \cap[n])}{n} .
$$

If each block of a partition has asymptotic frequency, this partition is said to have asymptotic frequencies. For $\pi \in \mathcal{P}_{\infty}$ possessing asymptotic frequencies, $|\pi|^{\downarrow}$ is the mass partition associated with $\pi$, that is, $\left(|\pi|_{i}^{\downarrow}\right)_{i \in \mathbb{N}}$ is the rearrangement in decreasing order of $\left(\left|\pi_{i}\right|\right)_{i \in \mathbb{N}}$. For every $n \in \mathbb{N}$, a permutation of $[n]$ is a bijection $\sigma:[n] \mapsto[n]$. For $n=\infty$, we define a permutation of $\mathbb{N}$ to be a bijection $\sigma$ of $\mathbb{N}$ such that $\sigma(k)=k$ when $k$ is large enough. We define the equivalence relation ' $\sim$ ' by $i \underset{\pi}{\sim} j$ if $i$ and $j$ are in the same block of $\pi$. We denote by $\sigma \pi$ the partition, defined by $\pi$

$$
i \underset{\sigma \pi}{\sim} j \Longleftrightarrow \sigma(i) \underset{\pi}{\sim} \sigma(j) .
$$

We stress that, due to the ranking of the blocks, $(\sigma \pi)_{i}=\sigma^{-1}\left(\pi_{\eta(i)}\right)$ for a certain permutation $\eta$.

A random partition $\pi$ of $\mathbb{N}$ is exchangeable if $\sigma \pi$ and $\pi$ have the same law for every permutation $\sigma$ of $\mathbb{N}$. Kingman established a correspondence between exchangeable partition laws and mass partitions via the paint-box partitions. We recall briefly the construction of 
paint boxes. Let $s$ be an element of $\mathcal{P}_{\mathrm{m}}$. Let $\mathcal{V}$ be an open subset of $(0,1)$ such that the ranked sequence of lengths of its interval components is given by $s$. Let $U_{1}, \ldots$ be an independent and identically distributed (i.i.d.) sequence of uniform variables on $[0,1]$. An $s$-paint box is the partition $\pi$ induced by the following equivalence relation: for all $i \neq j$,

$$
i \underset{\pi}{\sim} j \Longleftrightarrow U_{i} \text { and } U_{j} \text { belong to the same interval component of } \mathcal{V} \text {. }
$$

Kingman proved that any exchangeable partition is a mixture of paint boxes. We denote by $\rho_{s}$ the law of an $s$-paint box.

As explained in the introduction, we now extend this setting by distinguishing a block, working with partitions of $\mathbb{Z}_{+}$.

Definition 2.1. A distinguished partition $\pi$ is a partition of $\mathbb{Z}_{+}$where the block containing 0 is viewed as a distinguished block. Ranking the blocks in the order of their least element, the first block $\pi_{0}$ contains 0 and is the distinguished block of $\pi$.

We denote by $[n]$ the set $\{0,1, \ldots, n\}$, and by $\mathcal{P}_{n}^{0}$ the space of distinguished partitions of $\{0, \ldots, n\}$. For $n=\infty$, we agree that $[\infty]=\mathbb{Z}_{+}=\{0,1, \ldots\}$ and then $\mathcal{P}_{\infty}^{0}$ is the space of partitions of $\mathbb{Z}_{+}$. A first basic property is the compactness of the space $\mathcal{P}_{\infty}^{0}$ for the distance defined by

$$
d\left(\pi, \pi^{\prime}\right)=\left(1+\max \left\{n \geq 0, \pi_{\mid[n]}=\pi_{\mid[n]}^{\prime}\right\}\right)^{-1} .
$$

See [4] for a proof. Let $\pi \in \mathcal{P}_{n}^{0}$. For all $n^{\prime} \in \mathbb{Z}_{+} \cup\{\infty\}$ such that $n^{\prime} \geq n$, we define

$$
\mathcal{P}_{n^{\prime}, \pi}^{0}=\left\{\pi^{\prime} \in \mathcal{P}_{n^{\prime}}^{0} ; \pi_{\mid[n]}^{\prime}=\pi\right\} .
$$

A random distinguished partition is a random element of $\mathcal{P}_{\infty}^{0}$ equipped with the $\sigma$-field generated by the finite unions of the sets $\mathcal{P}_{n, \pi}^{0}$ (which corresponds to the Borelian $\sigma$-field for $d$ ).

In the same way, we introduce the set of distinguished mass partitions, meaning the sequences $s=\left(s_{i}\right)_{i \geq 0}$ of nonnegative real numbers such that $\sum_{i \geq 0} s_{i} \leq 1$, ranked in decreasing order apart from $s_{0}$ :

$$
\mathcal{P}_{\mathrm{m}}^{0}:=\left\{s=\left(s_{0}, s_{1}, \ldots\right) ; \sum_{i \geq 0} s_{i} \leq 1, s_{0} \geq 0, s_{1} \geq s_{2} \geq \cdots \geq 0\right\} .
$$

We identify the sets $\mathcal{P}_{\mathrm{m}}$ and $\left\{s \in \mathcal{P}_{\mathrm{m}}^{0} ; s_{0}=0\right\}$. The dust of $s$ is by definition the quantity $\delta:=1-\sum_{i=0}^{\infty} s_{i}$. A (distinguished) mass partition is said to be improper if the dust is positive. For $\pi \in \mathcal{P}_{\infty}^{0}$ having asymptotic frequencies, $|\pi|^{\downarrow}$ is the distinguished mass partition associated with $\pi$, that is, $|\pi|_{0}^{\downarrow}=\left|\pi_{0}\right|$ and $\left(|\pi|_{i}^{\downarrow}\right)_{i \in \mathbb{N}}$ is the rearrangement in decreasing order of $\left(\left|\pi_{i}\right|\right)_{i \in \mathbb{N}}$. We stress that, by definition, $|\pi|_{0}^{\downarrow}=\left|\pi_{0}\right|$.

We define a permutation of $\mathbb{Z}_{+}$to be a bijection $\sigma$ of $\mathbb{Z}_{+}$such that $\sigma(k)=k$ when $k$ is large enough. Note that any permutation of $\mathbb{N}$ can be extended to a permutation of $\mathbb{Z}_{+}$by deciding that $\sigma(0)=0$.

Definition 2.2. A random distinguished partition $\pi$ is exchangeable if $\sigma \pi$ and $\pi$ have the same law for every permutation $\sigma$ of $\mathbb{Z}_{+}$such that $\sigma(0)=0$.

It is easily seen that the restriction of an exchangeable distinguished partition to $\mathbb{N}$ is exchangeable. The converse may fail: there exist distinguished partitions which are not exchangeable though their restriction to $\mathbb{N}$ is exchangeable. We construct a counterexample: 
let $\pi$ be a nondegenerate exchangeable random partition and let $\pi^{0}$ be obtained from $\pi$ by distinguishing the block containing 1, i.e. $\pi^{0}=\left(\pi_{1} \cup\{0\}, \pi_{2}, \ldots\right)$ with blocks enumerated in order of appearance. The restriction $\pi_{\mid \mathbb{N}}^{0}=\pi$ is exchangeable. The structure of $\pi$ implies that $\mathrm{P}\left[\pi_{\mid[2]}^{0}=(\{0,1\},\{2\})\right]=\mathrm{P}\left[1 \chi_{\pi} 2\right]>0$. Let $\sigma$ be the permutation of $[2]: \sigma(0)=0$, $\sigma(1)=2, \sigma(2)=1$. We have $\mathrm{P}\left[\pi_{\mid[2]}^{0}=(\{0, \sigma(1)\},\{\sigma(2)\})\right]=\mathrm{P}\left[\pi_{1}=\{2\}\right]=0$. We have thus found a permutation such that $\mathrm{P}\left[\pi^{0}=\sigma\left(\pi_{0}^{0}, \ldots\right)\right] \neq \mathrm{P}\left[\pi^{0}=\left(\pi_{0}^{0}, \ldots\right)\right]$.

We define now the distinguished paint boxes and extend Kingman's correspondence to exchangeable distinguished partitions.

Definition 2.3. A distinguished paint box can be constructed in the following way. Let $s$ be a distinguished mass partition; we denote by $\delta$ its dust. Denote by $\partial$ an element which does not belong to $\mathbb{Z}_{+}$. Let $\xi$ be a probability on $\mathbb{Z}_{+} \cup\{\partial\}$ such that, for all $k \geq 0, \xi(k)=s_{k}$ and $\xi(\partial)=\delta$. Let $X_{1}, X_{2}, \ldots$ be a sequence of i.i.d. random variables with distribution $\xi$, and let $X_{0}=0$. An $s$-distinguished paint box is defined by, for all $i \neq j \geq 0$,

$$
\Pi^{0}: i \sim j \quad \Longleftrightarrow \quad X_{i}=X_{j} \neq \partial .
$$

In particular, $\Pi_{0}^{0}:=\left\{i \geq 0 ; X_{i}=0\right\}=\left\{i \geq 1 ; X_{i}=0\right\} \cup\{0\}$.

We denote by $\rho_{s}^{0}$ the law of an $s$-distinguished paint box. When $s_{0}=0$, the block $\Pi_{0}^{0}$ is the singleton $\{0\}$ and the $s$-distinguished paint box restricted to $\mathbb{N}$ is a classical $s$-paint-box partition of $\mathbb{N}$. According to the previous notation for paint boxes, we will denote its law by $\rho_{s}$.

Another way to define an $s$-distinguished paint box is to consider a subprobability $\alpha$ on $[0,1)$ and set $s=\left(\alpha(0), \alpha\left(x_{1}\right), \alpha\left(x_{2}\right), \ldots\right)$, where $x_{1}, x_{2}, \ldots$ are the atoms of $\alpha$ in $(0,1)$ ranked in decreasing order of their sizes. Let $X_{1}, X_{2}, \ldots$ be independent with law $\alpha$, and let $X_{0}=0$. The partition $\Pi^{0}$ defined by, for $i \neq j$,

$$
i \sim j \quad \Longleftrightarrow \quad X_{i}=X_{j}
$$

is an $s$-distinguished paint box.

Equivalently, we can work with uniform variables: an interval representation of $s$ is a collection of disjoint intervals $\left(A_{0}, A_{1}, A_{2}, \ldots\right)$, where $A_{0}$ is $\left[0, s_{0}\right]$ and $\left(A_{i}\right)_{i \geq 1}$ is such that the decreasing sequence of their lengths is $\left(s_{1}, s_{2}, \ldots\right)$. Draw an infinite sequence of uniform independent variables $\left(U_{i}\right)_{i \geq 1}$ and fix $U_{0}=0$. The partition of $\mathbb{Z}_{+}$defined by $\pi^{0}:=i \sim j$ if and only if $U_{i}$ and $U_{j}$ fall in the same interval (if $U_{i}$ falls in the dust of $s$ then $\{i\}$ is a singleton block of $\pi^{0}$ ) is an $s$-distinguished paint box. We stress that its law does not depend on the choice of intervals $\left(A_{1}, A_{2}, \ldots\right)$, and then we can choose $A_{i}:=\left[s_{0}+\cdots+s_{i-1}, s_{0}+\cdots+s_{i}\right)$ for all $i \geq 1$.

Proposition 2.1. Let $s \in \mathcal{P}_{\mathrm{m}}^{0}$, and let $\pi^{0}$ be an $s$-distinguished paint box.

(i) The distinguished paint box $\pi^{0}$ is exchangeable.

(ii) $\pi^{0}$ has asymptotic frequencies, and, more precisely, $\left|\pi^{0}\right|^{\downarrow}=s$.

(iii) For every $i \in \mathbb{Z}_{+}$, if $\left|\pi_{i}^{0}\right|=0$ then $\pi_{i}^{0}$ is a singleton or empty.

(iv) $s$ is improper if and only if some blocks different from $\pi_{0}^{0}$ are singletons. In this case the set of singletons $\left\{i \in \mathbb{Z}_{+}: i\right.$ is a singleton of $\left.\pi^{0}\right\}$ has an asymptotic frequency given by the dust $\delta=1-\sum_{i=0}^{\infty} s_{i}$ almost surely.

(v) We have $\rho_{s}^{0}(0$ is a singleton $)=0$ if $s_{0}>0$ and 1 otherwise, and $\rho_{s}^{0}(1,2, \ldots, q$ are singletons $)=\delta^{q}$ for $q \geq 1$. 
Proof. See the proof of Proposition 2.8 of [4].

It remains to see whether the distinguished paint-box construction of Definition 2.3 yields all the exchangeable distinguished partitions.

Theorem 2.1. Let $\pi^{0}$ be a random distinguished partition. The following assertions are equivalent:

(i) $\pi^{0}$ is exchangeable,

(ii) there exists a random distinguished mass partition $S=\left(S_{0}, S_{1}, \ldots\right)$ such that, conditionally given $S=s, \pi^{0}$ has the law of an $s$-distinguished paint box $\left(\rho_{s}^{0}\right)$. Furthermore, $\left|\pi^{0}\right|^{\downarrow}=S$.

Proof. A mixture of distinguished paint boxes is still exchangeable, proving that (ii) implies (i). Let $\pi^{0}$ be exchangeable. We adapt a proof of Aldous [1]; see also [4, p. 101]. We define a selection map to be any random function $b: \mathbb{Z}_{+} \rightarrow \mathbb{Z}_{+}$that maps all the points of the block $\pi_{0}^{0}$ to 0 , and all the points of a block $\pi_{i}^{0}$ for $i \geq 1$ to the same point of that block. Let $U_{0}=0,\left(U_{i}\right)_{i \geq 1}$ be i.i.d. uniform on [0,1], independent of $\pi^{0}$ and of the selection map $b$. We define $X_{n}=U_{b(n)}$. The law of $\left(X_{n}, n \geq 1\right)$ does not depend on the choice of $b$. The key of the proof is the exchangeability of $\left(X_{n}\right)_{n \geq 1}$. Let $\sigma$ be a permutation with $\sigma(0)=0$. We have

$$
X_{\sigma(n)}=U_{b(\sigma(n))}=U_{b^{\prime}(n)}^{\prime},
$$

where $U_{i}^{\prime}=U_{\sigma(i)}$ and $b^{\prime}=\sigma^{-1} \circ b \circ \sigma$. We verify that $b^{\prime}$ is a selection map for the partition $\sigma \pi^{0}$. Let $i \geq 0$ and $n \in \sigma \pi_{i}^{0}$, by the definition of $\sigma \pi^{0}, \sigma \pi_{i}^{0}=\sigma^{-1}\left(\pi_{\eta(i)}^{0}\right)$ for a certain permutation $\eta$ such that $\eta(0)=0$. Then there exists a $k \in \pi_{\eta(i)}^{0}$ such that $n=\sigma^{-1}(k)$. For $i=0$ and $k \in \pi_{0}^{0}, b^{\prime}(n)=\sigma^{-1}(b(k))=\sigma^{-1}(0)=0$ for all $n \in \sigma \pi_{0}^{0}$. For $i \geq 1$, we clearly find that $b^{\prime}(n)=\sigma^{-1} \circ b(k)$ depends only on $i$.

The sequence $\left(U_{i}^{\prime}, i \geq 1\right)$ has the same law as $\left(U_{i}, i \geq 1\right)$. By exchangeability and the independence of $\left(U_{j}\right)$ and $\pi^{0},\left(\left(U_{n}^{\prime}\right)_{n \geq 1}, \sigma \pi^{0}\right)$ has the same law as $\left(\left(U_{n}\right)_{n \geq 1}, \pi^{0}\right)$, and the sequence $\left(X_{n}, n \geq 1\right)$ is exchangeable. By the de Finetti theorem, conditionally on the random probability measure $\mu:=\lim _{n \rightarrow \infty}(1 / n) \sum_{i=1}^{n} \delta_{X_{i}},\left(X_{n}, n \geq 1\right)$ are i.i.d. random variables with distribution $\mu$. Moreover, by the definition of $X_{n}, i \underset{\pi}{\sim} j$ if and only if $X_{i}=X_{j}$. We deduce that, conditionally given $\mu=\alpha$, the distinguished partition $\pi^{0}$ is an $s(\alpha)$-distinguished paint box with $s(\alpha):=\left(\alpha(0), \alpha\left(x_{1}\right), \ldots\right)$. By the distinguished paint-box construction, on $\{\mu=\alpha\}, s(\alpha)$ is the mass partition of $\pi^{0}$ and so $\left|\pi^{0}\right|^{\downarrow}=s(\mu)$; moreover, the random sequence $S:=s(\mu)$ in $\mathcal{P}_{\mathrm{m}}^{0}$ verifies assertion (ii). To conclude, the random partition $\pi^{0}$ has the law as an $\eta$-mixture of distinguished paint boxes, where $\eta$ is the law of $\left|\pi^{0}\right|^{\downarrow}$. This completes the proof.

Theorem 2.1 sets up a bijection between probability distributions for exchangeable distinguished partitions and probability distributions on the space of distinguished mass partitions, $\mathcal{P}_{\mathrm{m}}^{0}$ :

$$
\mathrm{P}\left[\pi^{0} \in \cdot\right]=\int_{\mathcal{P}_{m}^{0}} \rho_{s}^{0}(\cdot) \mathrm{P}\left(\left|\pi^{0}\right|^{\downarrow} \in \mathrm{d} s\right) .
$$

Remark 2.1. Let $\pi^{0}$ be an exchangeable distinguished partition. As for exchangeable partitions (see [23, p. 43]), we can show that there exists a function $p$ such that

$$
\mathrm{P}\left[\pi_{\mid[n]}^{0}=\left(B_{0}, \ldots, B_{k}\right)\right]=p\left(n_{0}, \ldots, n_{k}\right),
$$

where $n_{i}=\# B_{i}, i=0, \ldots, k$. Contrary to exchangeable random partitions, the function $p$ is 
not totally symmetric but only invariant by permutations of the arguments $\left(n_{1}, \ldots, n_{k}\right)$. Indeed, by exchangeability, $\mathrm{P}\left[\pi^{0}=\left(\sigma B_{0}, \ldots, \sigma B_{k}\right)\right]=p\left(\# B_{0}, \ldots, \# B_{k}\right)=p\left(\# B_{\eta(0)}, \ldots, \# B_{\eta(k)}\right)$, where $\eta$ is the permutation such that $\sigma \pi_{i}^{0}=\sigma^{-1}\left(\pi_{\eta(i)}^{0}\right)$. Owing to the assumption that $\sigma(0)=$ 0 , the permutation $\eta$ is such that $\eta(0)=0$. The exchangeable distinguished partitions are thus special cases of partially exchangeable partitions in the sense of [21].

We mention that Donnelly and Joyce [12] defined the exchangeable ordered partitions for which all the blocks are distinguished (they speak about exchangeable random ranking). They obtained a Kingman representation for the exchangeable ordered partition structure. For every $\mu$ probability on $[0,1]$, an 'ordered paint box' is constructed from a sequence of i.i.d. $\mu$ variables. We stress that, contrary to an exchangeable distinguished paint box, the law of a $\mu$-ordered paint box depends on the order of the atoms of $\mu$. Exchangeable ordered partitions are also partially exchangeable but the function $p$ has no symmetry properties. Gnedin [15] gave a representation of exchangeable compositions which are a generalization of exchangeable random rankings.

We could define distinguished partitions with several distinguished blocks. It corresponds to a population with several sources of immigration: each distinguished block gathers the progeny of an immigration source. For the sake of simplicity, we distinguish here just one block.

In the next section we define distinguished coalescents which can be interpreted as a genealogy for a population with immigration.

\section{Distinguished coalescents}

Imagine an infinite haploid population with immigration. We denote by $\Pi^{0}(t)$ the partition of the current population into families having the same ancestor $t$ generations earlier. As explained in the introduction, individuals who have no ancestor in the population at generation $t$ form the distinguished block of $\Pi^{0}(t)$. Actually, individual 0 can be viewed as their common ancestor. When some individuals have the same ancestor at generation $t$, they have the same ancestor at any generation $t^{\prime} \geq t$. In terms of partitions, all integers in the same block of $\Pi^{0}(t)$ are in the same block of $\Pi^{0}\left(t^{\prime}\right)$ for any $t^{\prime} \geq t$. The collection of partitions $\left(\Pi^{0}(t)\right)_{t \geq 0}$ will be a coalescent process. To define these processes and go from an exchangeable distinguished partition to another coarser partition, we have to introduce the coagulation operator.

\subsection{Coagulation operator and distinguished coalescents}

To define the distinguished exchangeable coalescents, we need to define an operator on the space of distinguished partitions.

Definition 3.1. Let $\pi, \pi^{\prime} \in \mathcal{P}_{\infty}^{0}$. The partition $\operatorname{Coag}\left(\pi, \pi^{\prime}\right)$ is defined by $\operatorname{Coag}\left(\pi, \pi^{\prime}\right)_{i}=\pi_{i}^{\prime \prime}$, where $\pi_{i}^{\prime \prime}=\bigcup_{j \in \pi_{i}^{\prime}} \pi_{j}$. The partition $\operatorname{Coag}\left(\pi, \pi^{\prime}\right)$ is exactly the partition obtained by coagulating blocks of $\pi$ according to blocks of $\pi^{\prime}$.

We denote by $0_{[\infty]}$ the partition into singletons $\{\{0\},\{1\}, \ldots\}$, and by $1_{[\infty]}$ the trivial partition $\left\{\mathbb{Z}^{+}, \varnothing, \ldots\right\}$. Plainly, for all $n \geq 0, \operatorname{Coag}\left(\pi, \pi^{\prime}\right)_{\mid[n]}=\operatorname{Coag}\left(\pi_{\mid[n]}, \pi_{\mid[n]}^{\prime}\right)$ and, for all $\pi \in \mathcal{P}_{\infty}^{0}, \operatorname{Coag}\left(\pi, 0_{[\infty]}\right)=\pi$ and $\operatorname{Coag}\left(\pi, 1_{[\infty]}\right)=1_{[\infty]}$. Note that, however, we do not have $\operatorname{Coag}\left(\pi, \pi^{\prime}\right)_{\mid K}=\operatorname{Coag}\left(\pi_{\mid K}, \pi^{\prime}{ }_{\mid K}\right)$ for $K \subset \mathbb{N}$ in general.

Proposition 3.1. Let $\pi$ and $\pi^{\prime}$ be two independent exchangeable distinguished partitions. The distinguished partition $\operatorname{Coag}\left(\pi, \pi^{\prime}\right)$ is also exchangeable.

Proof. See the proof of Lemma 4.3 of [4]. 
The coagulation operator allows us to define distinguished coalescents which are Markovian processes valued in distinguished partitions of $\mathbb{N}$.

Definition 3.2. A Markov process $\Pi^{0}$ with values in $\mathcal{P}_{\infty}^{0}$ is called a distinguished coalescent if its semigroup is given as follows: the conditional law of $\Pi^{0}\left(t+t^{\prime}\right)$ given $\Pi^{0}(t)=\pi^{0}$ is the law of $\operatorname{Coag}\left(\pi^{0}, \pi^{\prime}\right)$, where $\pi^{\prime}$ is some exchangeable distinguished partition (whose law depends only on $\left.t^{\prime}\right)$. A distinguished coalescent is called standard if $\Pi^{0}(0)=0_{[\infty]}$.

The properties of the coagulation operator (see [4]), imply that a distinguished coalescent $\Pi^{0}$ fulfills the Feller property. Therefore, the process has a càdlàg version and is strong Markovian. Plainly, the random partition $\Pi_{\mid \mathbb{N}}^{0}(t)$ is exchangeable for all $t \geq 0$. However, we stress that in general the process $\left(\Pi_{\mid \mathbb{N}}^{0}(t), t \geq 0\right)$ is not an exchangeable coalescent and not even Markovian. We will give an example in Section 4.2.

For every $n \geq 1$, the restriction $\Pi_{\mid[n]}^{0}$ is a continuous-time Markov chain with a semigroup given by the operator Coag. Let $\pi \in \mathcal{P}_{n}^{0} \backslash\left\{0_{[n]}\right\}$. We denote by $q_{\pi}$ the jump rate of $\Pi_{\mid[n]}^{0}$ from $0_{[n]}$ to $\pi$ :

$$
q_{\pi}:=\lim _{t \rightarrow 0+} \frac{1}{t} \mathrm{P}_{0_{[n]}}\left[\Pi_{\mid[n]}^{0}(t)=\pi\right]
$$

An easy adaptation of the proof of Proposition 4.4 of [4] gives the existence and uniqueness of the distinguished coagulation measure.

Definition 3.3. The distinguished coagulation measure of $\Pi^{0}$ is the unique measure $\mu^{0}$ on $\mathcal{P}_{\infty}^{0}$ such that $\mu^{0}\left(\left\{0_{[\infty]}\right\}\right)=0$ and

$$
\mu^{0}\left(\mathcal{P}_{\infty, \pi}^{0}\right)=q_{\pi}
$$

for every $n \in \mathbb{Z}_{+}$and every partition $\pi \in \mathcal{P}_{n}^{0}$.

Moreover, the measure $\mu^{0}$ fulfills

$$
\mu^{0}\left(\pi \in \mathcal{P}_{\infty}^{0}: \pi_{\mid[n]} \neq 0_{[n]}\right)<\infty \quad \text { and } \quad \mu^{0} \text { is exchangeable. }
$$

Conversely, any measure fulfilling the previous conditions will be called a distinguished coagulation measure.

Let $\mu^{0}$ be a distinguished coagulation measure. We construct explicitly a distinguished coalescent process with coagulation measure (in the sense of Definition 3.3) $\mu^{0}$. Let $N$ be a Poisson measure with intensity $\mathrm{d} t \otimes \mu^{0}(\mathrm{~d} \pi)$. Let $N_{b}$ be the image of $N$ by the map $(t, \pi) \mapsto$ $\left(t, \pi_{\mid[b]}\right)$. Its intensity, denoted by $\mu_{b}^{0}$, is the image of $\mu^{0}$ by the previous map. We denote by $\left(t_{i}, \pi_{i}\right)$ the atoms of $N_{b}$, and define a process $\left(\Pi^{b}(t), t \geq 0\right)$ by the following recursion: for all $0 \leq t<t_{1}, \Pi^{b}(t)=0_{[b]}$ and, if $t_{i} \leq t<t_{i+1}, \Pi^{b}(t)=\operatorname{Coag}\left(\Pi^{b}\left(t_{i-1}\right), \pi^{i}\right)$, with $t_{0}=0$.

Proposition 3.2. The sequence of random partitions $\left(\Pi^{b}(t), b \in \mathbb{N}\right)$ is compatible, which means that, for all $a \leq b, \Pi_{\mid[a]}^{b}=\Pi^{a}$. The unique process $\left(\Pi^{0}(t), t \geq 0\right)$ such that $\Pi_{\mid[b]}^{0}(t)=\Pi^{b}(t)$, defined by $\Pi_{i}^{0}(t)=\bigcup_{b \geq 1} \Pi_{i}^{b}(t)$, is a distinguished coalescent with coagulation measure $\mu^{0}$.

Proof. The same arguments as those in the proof of Proposition 4.5 of [4] apply.

Example 3.1. We denote by $K(i, j)$ the simple distinguished partition where $i$ and $j$ are in the same block and all the other blocks are singletons. Let $c_{0}$ and $c_{1}$ be two nonnegative real numbers. The measure $\mu^{0}=c_{0} \mu_{0}^{K}+c_{1} \mu_{1}^{K}$, where $\mu_{0}^{K}:=\sum_{1 \leq i} \delta_{K(0, i)}$ and $\mu_{1}^{K}:=$ $\sum_{1 \leq i<j} \delta_{K(i, j)}$, is a distinguished coagulation measure. The process obtained is called Kingman's distinguished coalescent with rates $\left(c_{0}, c_{1}\right)$. 
Indeed, the measure $\mu^{0}$ defined as above is plainly a distinguished coagulation measure. The Poissonian construction explains the dynamics of this process. At a constant rate $c_{0}$, a block not containing 0 merges with $\Pi_{0}^{0}$ that is a singular coagulation with the distinguished block. At a constant rate $c_{1}$, two blocks not containing 0 merge into one, which is the classic binary coagulation of Kingman's coalescent.

\subsection{Characterization in law of the distinguished coalescents}

The next theorem is one of the main results of this work, it claims that the law of a distinguished coalescent is characterized by two nonnegative real numbers $c_{0}$ and $c_{1}$, and a measure $v^{0}$ on $\mathcal{P}_{\mathrm{m}}^{0}$. It should be viewed as an extension of Theorem 2.1 to certain infinite measures. Recall that $\rho_{s}^{0}$ denotes the law of an exchangeable distinguished $s$-paint box for $s \in \mathcal{P}_{\mathrm{m}}^{0}$.

Theorem 3.1. Recalling Definition 3.3, let $\mu^{0}$ be a distinguished coagulation measure. There exist two unique real numbers $c_{0}$ and $c_{1}$ and a unique measure $\nu^{0}$ on $\mathcal{P}_{\mathrm{m}}^{0}$ which satisfy

$$
\nu^{0}(0)=0 \text { and } \int_{\mathcal{P}_{\mathrm{m}}^{0}}\left(s_{0}+\sum_{i=1}^{\infty} s_{i}^{2}\right) v^{0}(\mathrm{~d} s)<\infty
$$

such that

$$
\mu^{0}=c_{0} \mu_{0}^{K}+c_{1} \mu_{1}^{K}+\rho_{\nu^{0}}^{0},
$$

where

$$
\rho_{\nu^{0}}^{0}(\cdot):=\int_{s \in \mathcal{P}_{\mathrm{m}}^{0}} \rho_{s}^{0}(\cdot) \nu^{0}(\mathrm{~d} s) .
$$

Conversely, let $c_{0}, c_{1}$, and $\nu^{0}$ be two real numbers and a measure on $\mathcal{P}_{\mathrm{m}}^{0}$ verifying the previous conditions. Then there exists a unique (in law) distinguished coalescent with $\mu^{0}=c_{0} \mu_{0}^{K}+$ $c_{1} \mu_{1}^{K}+\rho_{\nu^{0}}^{0}$.

When $\nu^{0}$ is carried on $\left\{s \in \mathcal{P}_{\mathrm{m}}^{0} ; s_{0}=0\right\}$ (which can be identified as $\mathcal{P}_{\mathrm{m}}$ ), the block containing 0 is reduced to the singleton $\{0\}$ (we distinguish no block), and considering the restriction to $\mathbb{N}$, we recover the characterization of exchangeable coalescents (also called $\Xi$-coalescents) in [26].

Proof of Theorem 3.1. Arguments used to prove this theorem are adapted from those of Theorem 4.2 of [4, Chapter 4]. Nevertheless, we give details to highlight the fact that the condition on $\nu^{0}$ differs from that of Theorem 4.2 of [4]. We denote by $\mu_{n}^{0}$ the restriction of $\mu^{0}$ to $\left\{\pi \in \mathcal{P}_{\infty}^{0} ; \pi_{\mid[n]} \neq 0_{[n]}\right\}$. The measure $\mu_{n}^{0}=\mathbf{1}_{\left\{\pi \in \mathcal{P}_{\infty}^{0} ; \pi_{[n]} \neq 0_{[n]}\right\}} \mu^{0}$ has a finite mass and is invariant under the action of permutations $\sigma$ that coincide with the identity on $[n]$. We define the $n$-shift on distinguished partitions by the map $\pi \rightarrow \pi^{\prime}$ defined by

$$
i \underset{\pi^{\prime}}{\sim} j \Longleftrightarrow i+n \underset{\pi}{\sim} j+n
$$

for all $i, j \geq 1$ and by

$$
0 \underset{\pi^{\prime}}{\sim} j \Longleftrightarrow 0 \underset{\pi}{\sim} j+n
$$

for all $j \geq 1$. The image of $\mu_{n}^{0}$ by the $n$-shift, denoted by $\bar{\mu}_{n}^{0}$, is invariant under the action of permutations $\sigma$ of $\mathbb{Z}_{+}$such that $\sigma(0)=0$. By Kingman's correspondence (Theorem 2.1),

$$
\bar{\mu}_{n}^{0}(\mathrm{~d} \pi)=\int_{\mathcal{P}_{\mathrm{m}}^{0}} \rho_{s}^{0}(\mathrm{~d} \pi) \bar{\mu}_{n}^{0}\left(|\pi|^{\downarrow} \in \mathrm{d} s\right) .
$$


Moreover, $\bar{\mu}_{n}^{0}$-almost every partition has asymptotic frequencies. The shift does not affect the asymptotic frequencies, and so $\mu_{n}^{0}$-almost every partition has asymptotic frequencies. The measure $\mu^{0}$ is the increasing limit of the $\mu_{n}^{0}$; we deduce that $\mu^{0}$-almost every partition has asymptotic frequencies.

By the distinguished paint-box representation of $\bar{\mu}_{n}^{0}$, we obtain, for all $s \in \mathcal{P}_{\mathrm{m}}^{0} \backslash\{0\}$,

$$
\mu_{n}^{0}\left(n+1 \sim n+2 \nsim 0 \text { or } 0 \sim n+\left.1|| \pi\right|^{\downarrow}=s\right)=s_{0}+\sum_{k=1}^{\infty} s_{k}^{2} .
$$

Let $v_{n}^{0}$ be the image of $\mu_{n}^{0}$ by the map $\pi \mapsto|\pi|^{\downarrow}$ :

$$
v_{n}^{0}(\mathrm{~d} s)=\mu_{n}^{0}\left(|\pi|^{\downarrow} \in \mathrm{d} s\right) .
$$

We stress that $v_{n}^{0}(\mathrm{~d} s)=\bar{\mu}_{n}^{0}\left(|\pi|^{\downarrow} \in \mathrm{d} s\right)$ because the $n$-shift has no impact on the asymptotic frequencies.

We have $\mu_{n}^{0}(n+1 \sim n+2 \nsim 0$ or $0 \sim n+1) \geq \int_{\mathcal{P}_{\mathrm{m}}^{0}}\left(s_{0}+\sum_{i=1}^{\infty} s_{i}^{2}\right) v_{n}^{0}(\mathrm{~d} s)$. Moreover, $\mu_{n}^{0}(n+1 \sim n+2 \not 0$ or $0 \sim n+1) \leq \mu^{0}(n+1 \sim n+2 \nsim 0$ or $0 \sim n+1)$.

By the exchangeability of $\mu^{0}$, $\mu^{0}(n+1 \sim n+2 \not 0$ or $0 \sim n+1)=\mu^{0}(1 \sim 2 \not 0$ or $0 \sim 1) \leq \mu^{0}\left(\pi_{\mid[2]} \neq 0_{[2]}\right)<\infty$.

We deduce that the finite measures $v_{n}^{0}$ increase as $n \uparrow \infty$ to the measure $\nu^{0}:=\mu^{0}\left(|\pi|^{\downarrow} \in \mathrm{d} s\right)$ and so

$$
\lim _{n \rightarrow \infty} \int_{\mathcal{P}_{\mathrm{m}}^{0}}\left(s_{0}+\sum_{i=1}^{\infty} s_{i}^{2}\right) \nu_{n}^{0}(\mathrm{~d} s)=\int_{\mathcal{P}_{\mathrm{m}}^{0}}\left(s_{0}+\sum_{i=1}^{\infty} s_{i}^{2}\right) \nu^{0}(\mathrm{~d} s) \leq \mu^{0}\left(\pi_{\mid[2]} \neq 0_{[2]}\right)<\infty .
$$

Let $k \in \mathbb{N}$ and $\pi^{[k]} \in \mathcal{P}_{k} \backslash\left\{0_{[k]}\right\}$. The sequence of events $\pi_{\mid\{k+1, \ldots, k+n\}} \neq 0_{\{k+1, \ldots, k+n\}}$ is increasing. Then we have

$$
\begin{aligned}
& \mu^{0}\left(\pi_{\mid[k]}=\pi^{[k]},|\pi|^{\downarrow} \neq 0\right) \\
& \quad=\lim _{n \rightarrow \infty} \mu^{0}\left(\pi_{\mid[k]}=\pi^{[k]},|\pi|^{\downarrow} \neq 0, \pi_{\mid\{k+1, \ldots, k+n\}} \neq 0_{\{k+1, \ldots, k+n\}}\right) .
\end{aligned}
$$

By an obvious permutation we obtain

$$
\mu^{0}\left(\pi_{\mid[k]}=\pi^{[k]},|\pi|^{\downarrow} \neq 0, \pi_{\mid\{k+1, \ldots, k+n\}} \neq 0_{\{k+1, \ldots, k+n\}}\right)=\bar{\mu}_{n}^{0}\left(\pi_{\mid[k]}=\pi^{[k]},|\pi|^{\downarrow} \neq 0\right) .
$$

Thus, using the distinguished paint-box representation of $\bar{\mu}_{n}^{0}$, we deduce that

$$
\begin{aligned}
\bar{\mu}_{n}^{0}\left(\pi_{\mid[k]}=\pi^{[k]},|\pi|^{\downarrow} \neq 0\right) & =\int_{\mathcal{P}_{\mathrm{m}}^{0}} \rho_{s}^{0}\left(\pi_{\mid[k]}=\pi^{[k]},|\pi|^{\downarrow} \neq 0\right) \nu_{n}^{0}(\mathrm{~d} s) \\
& \rightarrow \int_{\mathcal{P}_{\mathrm{m}}^{0}} \rho_{s}^{0}\left(\pi_{\mid[k]}=\pi^{[k]},|\pi|^{\downarrow} \neq 0\right) \nu^{0}(\mathrm{~d} s) \quad \text { as } n \rightarrow \infty .
\end{aligned}
$$

As $k$ is arbitrary, we get

$$
\mathbf{1}_{\{|\pi| \downarrow \neq 0\}} \mu^{0}(\mathrm{~d} \pi)=\int_{\mathcal{P}_{\mathrm{m}}^{0}} \rho_{s}^{0}(\mathrm{~d} \pi) \nu^{0}(\mathrm{~d} s) .
$$

It remains to study $\mathbf{1}_{\left\{|\pi|^{\downarrow}=0\right\}} \mu^{0}(\mathrm{~d} \pi)$. Consider now $\tilde{\mu}^{0}(\mathrm{~d} \pi):=\mathbf{1}_{\left\{0 \sim 1,|\pi|^{\downarrow}=0\right\}} \mu^{0}(\mathrm{~d} \pi)$, which 
has finite mass (because $\left.\mu^{0}(0 \sim 1)<\infty\right)$. We want to show that $\tilde{\mu}^{0}(\mathrm{~d} \pi)$ is proportional to $\delta_{K(0,1)}$, where $K(0,1)$ is the simple partition with $0 \sim 1$. Let $\tilde{\mu}_{2}^{0}(\mathrm{~d} \pi)$ be the image of $\tilde{\mu}^{0}$ by the 2 -shift. The measure $\tilde{\mu}_{2}^{0}(\mathrm{~d} \pi)$ is supported by $\left\{\pi \in \mathcal{P}_{\infty} ;|\pi|^{\downarrow}=0\right\}$ and is exchangeable with finite mass. By the distinguished paint-box construction, the only exchangeable partition with asymptotic frequencies $|\pi|^{\downarrow}=0$ is the partition into singletons $0_{[\infty]}$. Therefore, $\tilde{\mu}_{2}^{0}(\mathrm{~d} \pi)=c_{0} \delta_{\{\{0\},\{1\}, \ldots\}}$. We deduce that, for $\tilde{\mu}_{2}^{0}$-almost every $\pi^{\prime}, i \underset{\pi^{\prime}}{\chi} j$ for all $i \neq j$. From the definition of the 2 -shift, we find that, for $\tilde{\mu}^{0}$-almost every $\pi$,

$$
i+2 \underset{\pi}{\not} j+2 \text { for all } i, j \geq 1, i \neq j, \quad \text { and } 0 \underset{\pi}{\not} j+2 \text { for all } j \geq 1 \text {. }
$$

This implies that we have to consider only three possibilities:

$$
\tilde{\mu}^{0}(\mathrm{~d} \pi)=c_{0} \delta_{K(0,1)}, \quad \tilde{\mu}^{0}(0 \sim 1 \sim 2)>0, \quad \text { or } \quad \tilde{\mu}^{0}(2 \sim k)>0 \quad \text { for some } k \geq 3 .
$$

If $\tilde{\mu}^{0}(2 \sim k)>0$ for some $k \geq 3$, we find by exchangeability that $\tilde{\mu}^{0}(2 \sim k)=\tilde{\mu}^{0}(2 \sim 3)>0$. Moreover, the collection of sets $\{\{2 \sim n\}, n \geq 3\}$ is such that the intersection of two or more sets has a zero measure $\tilde{\mu}^{0}$ and so $\tilde{\mu}^{0}\left(\bigcup_{n \geq 3}\{2 \sim n\}\right)=\sum_{n \geq 3} \tilde{\mu}^{0}(2 \sim n) \leq \mu^{0}(0 \sim 1)$. It follows that $\mu^{0}(0 \sim 1)=\infty$. This is a contradiction because $\mu^{0}(0 \sim 1)<\infty$. If $\tilde{\mu}^{0}(0 \sim 1 \sim 2)>0$ then, by exchangeability, for all $n \geq 2, \tilde{\mu}^{0}(0 \sim 1 \sim n)=c_{0}>0$ and, by the same arguments, the same contradiction appears. We deduce that $\tilde{\mu}^{0}(\mathrm{~d} \pi)$ is equal to $c_{0} \delta_{K(0,1)}$. By exchangeability we have $\mathbf{1}_{\{0 \sim i,|\pi| \downarrow=0\}} \mu^{0}(\mathrm{~d} \pi)=c_{0} \delta_{K(0, i)}$. The measure $\mu^{0} \mathbf{1}_{\left\{|\pi| \downarrow=0, \pi_{0} \neq\{0\}\right\}}$ is carried on the simple partition $\pi$ such that $\pi_{0}$ is not a singleton; moreover, the collection of sets $\{\{0 \sim i\}, i \geq 1\}$ is such that the intersection of two sets has a zero measure. Therefore, we have

$$
\mathbf{1}_{\left\{|\pi|^{\downarrow}=0, \pi_{0} \neq\{0\}\right\}} \mu^{0}(\mathrm{~d} \pi)=c_{0} \sum_{i \geq 1} \delta_{K(0, i)} .
$$

The restriction of $\mu^{0}$ to $\left\{\pi \in \mathcal{P}_{\infty}^{0} ; \pi_{0}=\{0\}\right\}$ can be viewed as an exchangeable measure on $\mathcal{P}_{\infty}$; the argument to conclude is then the same as in [4, p. 184].

Remark 3.1. Denote by $\left(D_{0}(t)\right)_{t \geq 0}:=\left(1-\sum_{i=0}^{\infty}\left|\Pi_{i}^{0}(t)\right|\right)_{t \geq 0}$ the process of the dust of $\Pi^{0}$. The arguments of [22] or [4] allow us to show that, for all $t>0$, the random partition $\Pi^{0}(t)$ has improper asymptotic frequencies with a strictly positive probability if and only if $c_{1}=0$ and $\int_{\mathcal{P}_{\mathrm{m}}^{0}}(1-\delta) \nu^{0}(\mathrm{~d} s)<\infty$, where $\delta=1-\sum_{i=0}^{\infty} s_{i}$. In that case, the process $\left(\xi^{0}(t)\right)_{t \geq 0}:=$ $\left(-\ln \left(D_{0}(t)\right)\right)_{t \geq 0}$ is a subordinator with Laplace exponent

$$
\phi^{0}(q)=c_{0} q+\int_{\mathcal{P}_{\mathrm{m}}^{0}}\left(1-\delta^{q}\right) \nu^{0}(\mathrm{~d} s) .
$$

Note that the drift coefficient $c_{0}$ may be positive, which contrasts with the result of Pitman [22].

\section{The simple distinguished exchangeable coalescents: $M$-coalescents}

In this section we focus on simple distinguished coalescents for which the coagulation measure $\mu^{0}$ is carried by the set of simple distinguished partitions. We call them, hereafter, $M$-coalescents. These processes are the analogue of $\Lambda$-coalescents for exchangeable distinguished coalescents. Historically, the $\Lambda$-coalescent is the first exchangeable coalescent with multiple collisions to have been defined; see [22] and [24]. We begin by recalling some basic facts about $\Lambda$-coalescents. 


\section{1. $\Lambda$-coalescents}

A $\Lambda$-coalescent (also called a simple exchangeable coalescent) is a process taking values in the partitions of $\mathbb{N}$ describing the genealogy of an infinite haploid population, labelled by $\mathbb{N}$ where two or more ancestral lineages merging cannot occur simultaneously. We stress that in these coalescent processes, each individual has an ancestor in the population. Immigration phenomenon is not taken into account and no block is distinguished. A simple exchangeable coalescent is a Markovian process $(\Pi(t), t \geq 0)$ on the space of partitions of $\mathbb{N}$ satisfying the following conditions.

(i) If $n \in \mathbb{N}$ then the restriction $\left(\Pi_{\mid[n]}(t)\right)$ is a continuous-time Markov chain valued in $\mathcal{P}_{n}$.

(ii) For each $n,\left(\Pi_{\mid[n]}(t)\right)$ evolves by the exchangeable merging of blocks: $\Pi_{\mid[n]}(t)=$ $\operatorname{Coag}\left(\Pi_{\mid[n]}(t-), \pi^{\prime}\right)$, where $\pi^{\prime}$ is an independent simple exchangeable partition.

By Theorem 1 of [22], or [24], we know that any simple exchangeable coalescent is characterized in law by a finite measure $\Lambda$ on $[0,1]$. The dynamics of $\Pi$ can be described as follows. Whenever $\Pi_{\mid[n]}(t)$ is a partition with $b$ blocks, the rate at which a $k$-tuple of its blocks merges is

$$
\lambda_{b, k}=\int_{0}^{1} x^{k-2}(1-x)^{b-k} \Lambda(\mathrm{d} x) .
$$

When $\Lambda$ is the Dirac at 0 , we recover Kingman's coalescent. When $\Lambda(\{0\})=0$, the $\Lambda$ coalescent can be constructed via a Poisson point process on $\mathbb{R}_{+} \times[0,1]$ with intensity $\mathrm{d} t \otimes$ $v(\mathrm{~d} x)$, where $v(\mathrm{~d} x)=x^{-2} \Lambda(\mathrm{d} x)$ :

$$
N=\sum_{i \in \mathbb{N}} \delta_{\left(t_{i}, x_{i}\right)} .
$$

The atoms of $N$ encode the evolution of the coalescent $\Pi$. At time $t-$, flip a coin for which the probability of 'heads' is $x$ for each block. All blocks flipping 'heads' are merged immediately. We can also construct a simple exchangeable partition, $\pi^{\prime}$, where the nontrivial block is constituted by indices of 'heads'. Thus, we get $\Pi(t)$ by $\operatorname{Coag}\left(\Pi(t-), \pi^{\prime}\right)$. In order to make this construction rigorous, we first consider the restrictions $\left(\Pi_{\mid[n]}(t)\right)$ as in Proposition 3.2, since the measure $v(\mathrm{~d} x):=x^{-2} \Lambda(\mathrm{d} x)$ can have an infinite mass.

\section{2. $M$-coalescents}

The distinguished exchangeable coalescents such that when a coagulation occurs all the blocks involved merge as a single block are called $M$-coalescents. We specify their laws by two finite measures on $[0,1]$, and study their generators in the same fashion as those of $\Lambda$-coalescents.

Definition 4.1. When a distinguished coagulation measure $\mu^{0}$ is carried by the set of simple distinguished partitions (with only one block nonempty or a singleton), the distinguished coalescent $\Pi^{0}$ is said to be simple. Define the following restricted measures:

$$
v_{0}=v^{0} \mathbf{1}_{\left\{s \in \mathcal{P}_{\mathrm{m}}^{0} ; s=\left(s_{0}, 0, \ldots\right)\right\}} \quad \text { and } \quad v_{1}=v^{0} \mathbf{1}_{\left\{s \in \mathcal{P}_{\mathrm{m}}^{0} ; s=\left(0, s_{1}, 0, \ldots\right)\right\}} \cdot
$$

We can write $v^{0}=v_{0}+v_{1}$. By a slight abuse of notation, $v_{0}$ and $v_{1}$ can be viewed as two measures on $[0,1]$ such that $\int_{0}^{1} s_{0} v_{0}\left(\mathrm{~d} s_{0}\right)<\infty$ and $\int_{0}^{1} s_{1}^{2} v_{1}\left(\mathrm{~d} s_{1}\right)<\infty$, and Theorem 3.1 yields

$$
\mu^{0}=c_{0} \mu_{0}^{K}+\rho_{\nu_{0}}^{0}+c_{1} \mu_{1}^{K}+\rho_{\nu_{1}} .
$$


We define the finite measures $\Lambda_{0}(\mathrm{~d} x):=x v_{0}(\mathrm{~d} x)+c_{0} \delta_{0}$ and $\Lambda_{1}(\mathrm{~d} x):=x^{2} v_{1}(\mathrm{~d} x)+c_{1} \delta_{0}$. The law of a simple distinguished coalescent is then characterized by $M=\left(\Lambda_{0}, \Lambda_{1}\right)$, and we call this subclass the $M$-coalescents.

As already mentioned in Section 3, in most cases, the restriction to $\mathbb{N}$ of a distinguished coalescent is not Markovian. Let $\Pi^{0}$ be an $M$-coalescent with, for instance, $\Lambda_{0}=\delta_{0}$ and $\Lambda_{1}(\mathrm{~d} x)=\mathrm{d} x$ (the Lebesgue measure). To locate the distinguished block in $\Pi_{\mid \mathbb{N}}^{0}$, we may locate a binary coagulation before time $t$ (all other mergers involve an infinite number of blocks). The restricted process $\Pi_{\mid \mathbb{N}}^{0}$ is then not Markovian.

The explicit Poissonian construction of Proposition 3.2 can now be interpreted in the same way as that of $\Lambda$-coalescents; see Section 4.1. When $\Lambda_{0}(\{0\})=\Lambda_{1}(\{0\})=0$, the $M$-coalescent associated can be constructed via two Poisson point processes $N_{0}$ and $N_{1}$ on $\mathbb{R}_{+} \times(0,1]$ with intensities $\mathrm{d} t \otimes v_{0}(\mathrm{~d} x)$ and $\mathrm{d} t \otimes v_{1}(\mathrm{~d} x)$, where $v_{0}(\mathrm{~d} x)=x^{-1} \Lambda_{0}(\mathrm{~d} x)$ and $v_{1}(\mathrm{~d} x)=$ $x^{-2} \Lambda_{1}(\mathrm{~d} x)$.

- At an atom $\left(t_{i}, x_{i}\right)$ of $N_{1}$, flip a coin for which the probability of 'heads' is $x_{i}$ for each block not containing 0 . All blocks flipping 'heads' are merged immediately into one block as in the Proposition 3.2.

- At an atom $\left(t_{i}, x_{i}\right)$ of $N_{0}$, flip a coin for which the probability of 'heads' is $x_{i}$ for each block not containing 0 . All blocks flipping 'heads' coagulate immediately with the distinguished block.

This construction is exactly that obtained when we coagulate the partition at $t$ - with a simple exchangeable distinguished partition $\pi^{\prime}$ where the nontrivial block is constituted by indexes of 'heads'. Thus, we construct the $M$-coalescent in the same way as the $\Lambda$-coalescent in Section 4.1.

We investigate jump rates of an $M$-coalescent $\left(\Pi^{0}(t)\right)_{t \geq 0}$. Thanks to the simple distinguished paint-box structure, we compute explicitly the jump rates of the restriction of $\Pi^{0}$. Let $\pi \in \mathcal{P}_{n}^{0}$ be simple, $q_{\pi}=\mu^{0}\left(\mathcal{P}_{\infty, \pi}^{0}\right)$.

- For every $2 \leq k \leq n$, if $\pi$ has one block not containing 0 with $k$ elements then

$$
q_{\pi}=\lambda_{n, k}:=\int_{0}^{1} x^{k-2}(1-x)^{n-k} \Lambda_{1}(\mathrm{~d} x)
$$

- For every $1 \leq k \leq n$, if the distinguished block of $\pi$ has $k+1$ elements (counting 0 ) then

$$
q_{\pi}=r_{n, k}:=\int_{0}^{1} y^{k-1}(1-y)^{n-k} \Lambda_{0}(\mathrm{~d} y) .
$$

Let $\pi \in \mathcal{P}_{p}^{0}$ with $b$ blocks without 0 , and let $F$ be any function defined on $\mathcal{P}_{p}^{0}$. The generator of $\Pi_{\mid[p]}^{0}$ is

$$
\mathcal{L}^{*} F(\pi)=\sum_{I \subset\{1, \ldots, b\},|I| \geq 2} \lambda_{b,|I|}\left(F\left(c_{I} \pi\right)-F(\pi)\right)+\sum_{J \subset\{1, \ldots, b\},|J| \geq 1} r_{b,|J|}\left(F\left(c^{J} \pi\right)-F(\pi)\right),
$$

with $c_{I} \pi=\operatorname{Coag}(\pi,\{\{0\},\{1\}, \ldots,\{I\}, \ldots\})$ and $c^{J} \pi=\operatorname{Coag}(\pi,\{\{0\} \cup\{J\},\{\cdot\}, \ldots,\{\cdot\}\})$. 


\subsection{Coming down from infinity for $M$-coalescents}

Let $\Lambda$ be a finite measure on $[0,1]$, and let $\Pi$ be a $\Lambda$-coalescent. Pitman [22] showed that if $\Lambda(\{1\})=0$, only the following two types of behaviour are possible: either P[for all $t>0, \Pi(t)$ has infinitely many blocks] $=1$ or $\mathrm{P}[$ for all $t>0, \Pi(t)$ has only finitely many blocks $]=1$. In the second case, the process $\Pi$ is said to come down from infinity. For instance, Kingman's coalescent comes down from infinity, while if $\Lambda(\mathrm{d} x)=\mathrm{d} x$ then the corresponding $\Lambda$-coalescent (called the Bolthausen-Sznitman coalescent) does not come down from infinity. A necessary and sufficient condition for a $\Lambda$-coalescent to come down from infinity was given by Schweinsberg [25]. Define

$$
\phi(n)=\sum_{k=2}^{n}(k-1) C_{n}^{k} \lambda_{n, k}
$$

with $\lambda_{n, k}=\int_{0}^{1} x^{k-2}(1-x)^{n-k} \Lambda(\mathrm{d} x)$. The $\Lambda$-coalescent comes down from infinity if and only if $\sum_{n=2}^{\infty} 1 / \phi(n)<\infty$.

Define $\psi_{\Lambda}(q):=\int_{[0,1]}\left(\mathrm{e}^{-q x}-1+q x\right) x^{-2} \Lambda(\mathrm{d} x)$. Bertoin and Le Gall [7] observed that

$$
\sum_{n=2}^{\infty} \frac{1}{\phi(n)}<\infty \Longleftrightarrow \int_{a}^{\infty} \frac{\mathrm{d} q}{\psi_{\Lambda}(q)}<\infty,
$$

where the right-hand side holds for some $a>0$ (and then necessarily for all). This equivalence is explained in a probabilistic way by Berestycki et al. [2]; see also [3, p. 107].

As for the $\Lambda$-coalescent, if the $M$-coalescent comes down from infinity, it does so immediately.

Proposition 4.1. Let $\left(\Pi^{0}(t)\right)_{t \geq 0}$ be an $M$-coalescent, with $\Lambda_{0}$ and $\Lambda_{1}$ without mass at 1 . We denote by $T$ its time of coming down from infinity: $T=\inf \{t>0, \# \Pi(t)<\infty\}$. We have, almost surely, $T=0$ or $T=\infty$.

Proof. See the proof of Lemma 31 of [26].

We stress that, when $\Lambda_{0}+\Lambda_{1}$ has a mass at 1 , the $M$-coalescent comes down from infinity. Indeed, by the Poisson construction, in an exponential time $\tau$ of parameter $\left(\Lambda_{0}+\Lambda_{1}\right)(\{1\})$, the Poisson measure $N$ has an atom $\pi$ such that $\pi_{0}=\mathbb{Z}_{+}$or $\pi_{1}=\mathbb{N}$. Thus, for large $t$, the process $\Pi^{0}(t)$ has just one block.

It remains to focus on the case where $\Lambda_{0}+\Lambda_{1}$ has no mass at 1 . Intuitively, when the genuine $\Lambda_{1}$-coalescent comes down, all blocks merged into one in an almost surely finite time. On the one hand, we can think that the $\left(\Lambda_{0}, \Lambda_{1}\right)$-coalescent has more jumps and coagulates all its blocks faster. On the other hand, the perturbation due to the coagulation with the distinguished block on the general term of the sum, studied initially by Schweinsberg [25], is not sufficient to induce its convergence and so the coming down. The $\left(\Lambda_{0}, \Lambda_{1}\right)$-coalescent comes down from infinity if and only if the $\Lambda_{1}$-coalescent comes down.

Theorem 4.1. The $\left(\Lambda_{0}, \Lambda_{1}\right)$-coalescent comes down from infinity if and only if

$$
\sum_{n=2}^{\infty} \frac{1}{\phi_{1}(n)}<\infty
$$

where $\phi_{1}(n)=\sum_{k=2}^{n}(k-1) C_{n}^{k} \lambda_{n, k}$ and $\lambda_{n, k}$ is as in Section 4.2.

The proof requires rather technical arguments and is given in Section 6. 


\section{M-coalescents and generalized Fleming-Viot processes with immigration}

In this section we are interested in a population model which has exactly a genealogy given by an $M$-coalescent. A powerful method to study simultaneously the population model and its genealogy is to define some stochastic flows as Bertoin and Le Gall [5]. A process valued in the space of probability measures on $[0,1],\left(Z_{t}^{0}, t \geq 0\right)$, is embedded in the flow. The atoms of the random probability $Z_{t}^{0}$ represent the current types of frequency in the population at time $t$. Moreover, $Z_{t}^{0}$ has a distinguished atom at 0 representing the fraction of immigrants in the population. This process will be called the $M$-generalized Fleming-Viot process with immigration. Following [5], we begin by establishing a correspondence between some stochastic flows and $M$-coalescents.

\subsection{Stochastic flows of distinguished bridges}

By assumption, at any time the families describing the population form a distinguished exchangeable partition. Theorem 2.1 ensures that it has a distinguished paint-box structure. We have to study some random functions called distinguished bridges.

5.1.1. Distinguished bridges and exchangeable distinguished partitions. Considering the underlying law on $[0,1]$ associated with an $s$-distinguished paint box (see Definition 2.3), we introduce the distinguished bridges defined by

$$
b_{s}(r)=s_{0}+\sum_{i=1}^{\infty} s_{i} \mathbf{1}_{\left\{V_{i} \leq r\right\}}+\delta r,
$$

where $s$ is a distinguished mass partition and $\left(V_{i}\right)_{i \geq 1}$ is a sequence of independent uniform variables; see Figure 1. Let $U_{0}=0$ and $\left(U_{i}\right)_{i \geq 1}$ be an independent sequence of i.i.d. uniform variables. The partition given by $i \sim j$ if and only if $b_{s}^{-1}\left(U_{i}\right)=b_{s}^{-1}\left(U_{j}\right)$ is exactly the $s$-distinguished paint box. When $s_{0}=0$, the bridge encodes a paint-box partition with no distinguished block.

Focusing on $M$-coalescents, we need to focus only on two types of distinguished bridges:

- bridges with distinguished mass partition $(0, x, 0, \ldots): b_{0, x}(r)=x \mathbf{1}_{\{V \leq r\}}+r(1-x)$,

- bridges with distinguished mass partition $(x, 0,0, \ldots): b_{x, 0}(r)=x+r(1-x)$.

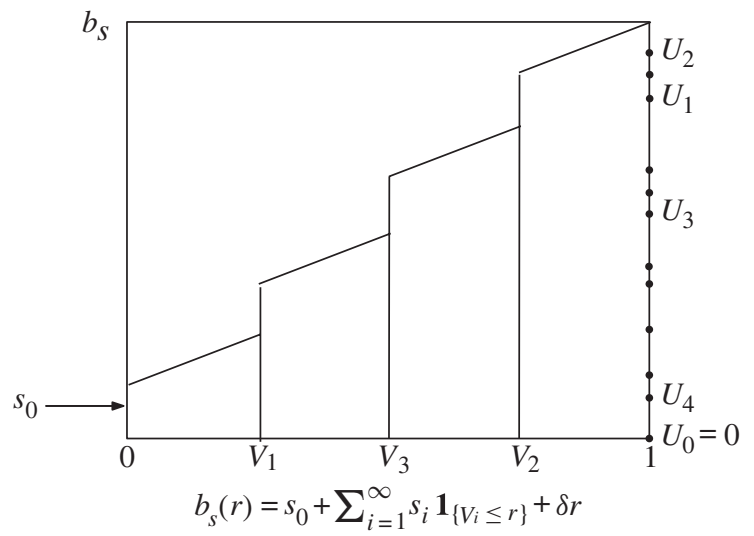

FIGURE 1: Distinguished bridge. 
To be concise, we will work directly with distinguished bridges of the form

$$
b_{y, x}(r)=y+x \mathbf{1}_{\{V \leq r\}}+r(1-x-y) .
$$

The following lemma relates the composition of distinguished bridges to the coagulation of simple distinguished partitions.

Lemma 5.1. Let $x, x^{\prime}, y, y^{\prime} \in[0,1]$ with $x+y, x^{\prime}+y^{\prime} \leq 1$, and let $b_{y, x}$ and $b_{y^{\prime}, x^{\prime}}$ be two independent distinguished bridges.

Let $\pi: i \sim j$ if and only if $b_{y, x}^{-1}\left(U_{i}\right)=b_{y, x}^{-1}\left(U_{j}\right)$. We stress that $\pi$ has at most two nontrivial blocks.

(i) For $i \geq 1$, we set $U_{i}^{\prime}:=b_{y, x}^{-1}\left(U_{j}\right)$ for all $j \in \pi_{i}$. The variables $\left(U_{i}^{\prime}\right)_{i \geq 1}$ are i.i.d. uniform, independent of $\pi$.

(ii) Let $\pi^{\prime}$ be the partition constructed from $b_{y^{\prime}, x^{\prime}}$ and $\left(U_{i}^{\prime}\right)_{i \geq 1}$. We denote by $\pi^{0}$ the partition such that $i \sim j$ if and only if $b_{y, x}^{-1} \circ b_{y^{\prime}, x^{\prime}}^{-1}\left(U_{i}\right)=b_{y, x}^{-1} \circ b_{y^{\prime}, x^{\prime}}^{-1}\left(U_{j}\right)$. We have the identity $\pi^{0}=\operatorname{Coag}\left(\pi, \pi^{\prime}\right)$.

Proof. The proof is an easy adaptation of Lemma 4.8 of [4].

Lemma 5.1 is the key observation in order to associate a stochastic flow of distinguished bridges, as defined below, with $M$-coalescents.

Definition 5.1. A flow of distinguished bridges is a collection $\left(B_{s, t},-\infty<s \leq t<\infty\right)$ of distinguished bridges such that

- for all $s<t<u, B_{s, u}=B_{s, t} \circ B_{t, u}$ almost surely,

- the law of $B_{s, t}$ depends only on $t-s$, and, for any $s_{1}<\cdots<s_{n}, B_{s_{1}, s_{2}}, \ldots, B_{s_{n-1}, s_{n}}$ are independent,

- $B_{0,0}=\mathrm{Id}$ and $B_{0, t} \rightarrow \mathrm{Id}$ in probability when $t \rightarrow 0$.

5.1.2. Poissonian construction of distinguished flows encoding $M$-coalescents. Let $M^{0}$ and $M^{1}$ be two independent Poissonian measures on $\mathbb{R} \times[0,1]$ with intensities $\mathrm{d} t \otimes v_{0}(\mathrm{~d} x)$ and $\mathrm{d} t \otimes$ $v_{1}(\mathrm{~d} x)$. We suppose that $v_{0}([0,1])+v_{1}([0,1])<\infty$ so that $N_{s, t}:=\left(M^{0}+M^{1}\right)((s, t] \times[0,1])$ is finite and $\left(N_{t}\right)=\left(N_{0, t}\right)_{t \geq 0}$ is a Poisson process. Let $\left(t_{i}^{0}, x_{i}^{0}\right)$ and $\left(t_{i}^{1}, x_{i}^{1}\right)$ be the atoms of $M^{0}$ and $M^{1}$ in $(s, t] \times[0,1]$. We define $B_{s, t}=b_{x_{1}} \circ \cdots \circ b_{x_{K}}$, where $K=N_{s, t}$ and $b_{x}$ denotes $b_{0, x}$ or $b_{x, 0}$ depending on whether $x$ is an atom of $M^{0}$ or $M^{1}$. From the independence of $M^{0}$ and $M^{1}$ and the independence of $M^{i}(A)$ and $M^{i}(B), i=0,1$, for $A$ and $B$ disjoint, $\left(B_{s, t}\right)_{s \leq t}$ is a flow in the sense of Definition 5.1.

Proposition 5.1. The process $\left(\Pi^{0}(t), t \geq 0\right)$ defined by $\Pi^{0}(t): i \sim j \Leftrightarrow B_{0, t}^{-1}\left(U_{i}\right)=$ $B_{0, t}^{-1}\left(U_{j}\right)$, where $B_{0, t}=b_{x_{1}} \circ \cdots \circ b_{x_{N_{t}}}$, is an M-coalescent with $M=\left(x v_{0}(\mathrm{~d} x), x^{2} v_{1}(\mathrm{~d} x)\right)$.

Proof. Lemma 5.1 implies that the process $\left(\Pi^{0}(t)\right)_{t \geq 0}$ corresponds to that built explicitly in Proposition 3.2.

The next result defines stochastic flows for general measures $v_{0}$ and $v_{1}$ on $[0,1]$.

Theorem 5.1. Let $\left(v_{0}^{n}\right)$ and $\left(v_{1}^{n}\right)$ be two sequences of finite measures on $[0,1]$. We call $\left(B_{s, t}^{(n)},-\infty<s \leq t<\infty\right)$ the associated flow of bridges. Assume the weak convergences 
of $\Lambda_{1}^{n}(\mathrm{~d} x):=x^{2} v_{1}^{n}(\mathrm{~d} x)$ to $\Lambda_{1}(\mathrm{~d} x):=c_{1} \delta_{0}(\mathrm{~d} x)+x^{2} v_{1}(\mathrm{~d} x)$ and $\Lambda_{0}^{n}(\mathrm{~d} y):=y v_{0}^{n}(\mathrm{~d} y)$ to $\Lambda_{0}(\mathrm{~d} y):=c_{0} \delta_{0}+y \mu(\mathrm{d} y)$. We obtain

- $\left(B_{s, t}^{(n)}, \infty<s \leq t<\infty\right)$ converges, in the sense of convergence of finite-dimensional distributions, to $\left(B_{s, t}, \infty<s \leq t<\infty\right)$, a stochastic flow,

- the process $\left(\Pi^{0}(t), t \geq 0\right)$ defined by $\Pi^{0}(t): i \sim j \Leftrightarrow B_{0, t}^{-1}\left(U_{i}\right)=B_{0, t}^{-1}\left(U_{j}\right)$ is an $M$-coalescent with rates $\left(\Lambda_{0}, \Lambda_{1}\right)$.

Proof. We denote by $\Pi^{(n)}(s, t)$ the random partition encoded by $B_{s, t}^{(n)}$. Under the previous assumptions on $v_{0}$ and $v_{1}$, the jump rates

$$
\lambda_{b, k}^{(n)}=\int_{0}^{1} x^{k}(1-x)^{b-k} v_{1}^{n}(\mathrm{~d} x) \quad \text { and } \quad r_{b, k}^{(n)}=\int_{0}^{1} y^{k}(1-y)^{b-k} v_{0}^{n}(\mathrm{~d} y)
$$

converge:

$$
\begin{aligned}
& \lambda_{b, k}^{(n)} \rightarrow c_{1} \mathbf{1}_{\{k=2\}}+\int_{0}^{1} x^{k}(1-x)^{b-k} \nu_{1}(\mathrm{~d} x):=\lambda_{b, k} \quad \text { as } n \rightarrow \infty, \\
& r_{b, k}^{(n)} \rightarrow c_{0} \mathbf{1}_{\{k=1\}}+\int_{0}^{1} x^{k}(1-x)^{b-k} \nu_{0}(\mathrm{~d} x):=r_{b, k} \quad \text { as } n \rightarrow \infty .
\end{aligned}
$$

The sequence of Markov chains $\left(\Pi_{\mid[k]}^{(n)}(s, t)\right)_{t \geq s}$ converges in the sense of finite-dimensional distributions to a distinguished coalescent chain, say $\Pi_{[[k]}^{0}(s, t)_{t \geq s}$. By compatibility, this implies the convergence of finite-dimensional distributions of $\left(\Pi^{(n)}(s, t)\right)_{n \geq 1}$ to $\Pi^{0}(s, t)$. According to Proposition 2.9 and Lemma 4.7 of [4] (which are easily adapted to our setting), we obtain the convergence of the distinguished mass partitions

$$
\left|\Pi^{(n)}(s, t)\right|^{\downarrow} \rightarrow\left|\Pi^{0}(s, t)\right|^{\downarrow} \quad \text { as } n \rightarrow \infty
$$

and the convergence of the bridge $B_{s, t}^{(n)}$ (which has jumps of size $\left|\Pi^{(n)}(s, t)\right|^{\downarrow}$ ) to a bridge $B_{s, t}$ (which has jumps of size $\left|\Pi^{0}(s, t)\right|^{\downarrow}$ ) for all $s, t \geq 0$ fixed. Thanks to the independence of $B_{s_{1}, s_{2}}^{(n)}, \ldots, B_{s_{k-1}, s_{k}}^{(n)}$ for any $s_{1}<\cdots<s_{k}$ and the flow property $\left(B_{s, t}^{(n)} \circ B_{t, u}^{(n)}=B_{s, u}^{(n)}\right)$, the one-dimensional convergence in distribution readily extends to finite-dimensional distributions. The existence of the flow $B$ is ensured by Kolmogorov's extension theorem.

Remark 5.1. As mentioned in Section 2 , we could define coalescents with several distinguished blocks. In particular, considering distinguished bridges which jump at 0 and 1 , we get a flow coding a coalescent with two distinguished blocks and a population with two immigration sources.

The composition of two distinguished bridges may be interpreted as the succession of two events (reproduction or immigration) in the population. A duality method provides a continuous population model.

\subsection{The dual distinguished flow and a population model with immigration}

In the same spirit of [4] and [5], we interpret the dual flow, $\left(\hat{B}_{s, t}\right):=\left(B_{-t,-s}\right)$, in terms of a natural model population on $[0,1)$ with fixed size 1 . We denote by $Z_{t}^{0}(\mathrm{~d} r)$, the random Stieljes measure of $\hat{B}_{0, t}$, i.e. $Z_{t}^{0}=\mathrm{d} \hat{B}_{0, t}$; it defines a Markov process with values in the space of probability measures on $[0,1)$ (denoted by $\mathcal{M}_{1}$ ). We may think of $Z_{t}^{0}(\mathrm{~d} r)$ and $Z_{t}^{0}(\{0\})$ respectively as the size of the progeny at time $t$ of the fraction $\mathrm{d} r$ of the initial population, and as the size at time $t$ of the immigrants descendants. 
The cocycle identity $\hat{B}_{t, u} \circ \hat{B}_{s, t}=\hat{B}_{s, u}$ ensures that $\left(Z_{t}^{0}\right)$ is a continuous-time Markov chain with the following dynamics, whenever the measures $v_{0}$ and $v_{1}$ are finite: if $t$ is a jump time for $Z_{\text {. }}^{0}$ then the conditional law of $Z_{t}^{0}$ given $Z_{t-}^{0}$ is that of

- $(1-X) Z_{t-}^{0}+X \delta_{U}$ if $t$ is an atom of $M^{1}$, where $X$ is distributed as $v_{1}(\cdot) / v_{1}([0,1])$ and $U$ as $Z_{t-}^{0}$,

- $(1-Y) Z_{t-}^{0}+Y \delta_{0}$ if $t$ is an atom of $M^{0}$, where $Y$ is distributed as $v_{0}(\cdot) / \nu_{0}([0,1])$.

At a reproduction time (meaning an atom of $M^{1}$ ) an individual picked at random in the population at generation $t$ - generates a proportion $X$ of the population at time $t$, as for the genuine generalized Fleming-Viot. At an immigration time (meaning an atom of $M^{0}$ ) the individual 0 at the time $t-$ generates a proportion $Y$ of the population at time $t$. In both cases, the rest of the population at time $t-$ is reduced by a factor $1-X$ or $1-Y$ so that, at time $t$, the total size is still 1 . We call this measure-valued process a generalized Fleming-Viot process with immigration (GFVI). The genealogy of this population (which is identified as $[0,1])$ coincides with an $M$-coalescent. Plainly, the generator of $\left(Z_{t}^{0}, t \geq 0\right)$ is

$$
\begin{aligned}
\mathcal{L} G(\rho)= & \int v_{1}(\mathrm{~d} x) \int \rho(\mathrm{d} a)\left[G\left((1-x) \rho+x \delta_{a}\right)-G(\rho)\right] \\
& +\int \nu_{0}(\mathrm{~d} y)\left[G\left((1-y) \rho+y \delta_{0}\right)-G(\rho)\right] .
\end{aligned}
$$

Thus, for any bounded function $G$ on $\mathcal{M}_{1}$, the space of probability measures on [0, 1],

$$
\begin{aligned}
& G\left(Z_{t}^{0}\right)-\int_{0}^{t} \mathrm{~d} s \int v_{1}(\mathrm{~d} x) \int Z_{s}^{0}(\mathrm{~d} a)\left[G\left((1-x) Z_{s}^{0}+x \delta_{a}\right)-G\left(Z_{s}^{0}\right)\right] \\
& -\int_{0}^{t} \mathrm{~d} s \int v_{0}(\mathrm{~d} y)\left[G\left((1-y) Z_{s}^{0}+y \delta_{0}\right)-G\left(Z_{s}^{0}\right)\right]
\end{aligned}
$$

is a martingale. Considering functions of the form

$$
G_{f}: \rho \in \mathcal{M}_{1} \mapsto \int_{[0,1] p} f\left(x_{1}, \ldots, x_{p}\right) \rho\left(\mathrm{d} x_{1}\right) \cdots \rho\left(\mathrm{d} x_{p}\right)=\left\langle f, \rho^{\otimes p}\right\rangle
$$

for $f$ a continuous function on $[0,1]^{p}$, we generalize in the following lemma this result for infinite measures.

Lemma 5.2. Assume that $v_{0}$ and $v_{1}$ have infinite masses, and that $c_{0}$ and $c_{1}$ are 0 . We define the operator $\mathcal{L}$, acting on functions of the type $G_{f}$, by

$$
\begin{aligned}
\mathcal{L} G_{f}(\rho)= & \int v_{1}(\mathrm{~d} x) \int \rho(\mathrm{d} a)\left[G_{f}\left((1-x) \rho+x \delta_{a}\right)-G_{f}(\rho)\right] \\
& +\int v_{0}(\mathrm{~d} y)\left[G_{f}\left((1-y) \rho+y \delta_{0}\right)-G_{f}(\rho)\right] .
\end{aligned}
$$

The process $G_{f}\left(Z_{t}^{0}\right)-\int_{0}^{t} \mathcal{L} G_{f}\left(Z_{s}^{0}\right) \mathrm{d} s$ is a martingale.

Proof. Consider two sequences $\left(v_{1}^{n}\right)$ and $\left(v_{0}^{n}\right)$ of finite measures on $[0,1)$. Suppose that $\Lambda_{1}^{n}(\mathrm{~d} x):=x^{2} \nu_{1}^{n}(\mathrm{~d} x)$ and $\Lambda_{0}^{n}(\mathrm{~d} x):=x v_{0}^{n}(\mathrm{~d} x)$ weakly converge to some finite measures $\Lambda_{1}(\mathrm{~d} x)$ and $\Lambda_{0}(\mathrm{~d} x)$. 
For $G_{f}(\rho)=\prod_{i}^{p}\left\langle\rho, \phi_{i}\right\rangle$, Bertoin and Le Gall [5] obtained

$$
\begin{aligned}
\int v_{1}^{n}(\mathrm{~d} x) \int \rho(\mathrm{d} a)\left[G\left((1-x) \rho+x \delta_{a}\right)-G(\rho)\right] \\
\quad=\sum_{\substack{I \subset\{1, \ldots, p\} \\
|I| \geq 2}} \lambda_{p,|I|}^{n} \int \rho\left(\mathrm{d} x_{1}\right) \cdots \rho\left(\mathrm{d} x_{p}\right)\left[f\left(x_{1}^{I}, \ldots, x_{p}^{I}\right)-f\left(x_{1}, \ldots, x_{p}\right)\right]
\end{aligned}
$$

with $\left(x_{1}^{I}, \ldots, x_{p}^{I}\right)=\left(y_{1}, \ldots, y_{p}\right)$, where, for all $i \in I, y_{i}=x_{\text {inf } I}$ and the values $y_{i}, i \notin I$, listed in the order of $\{1, \ldots, p\} \backslash I$ are the numbers $x_{1}, \ldots, x_{\text {inf } I-1}, x_{\text {inf } I+1}, \ldots, x_{p-|I|+1}$.

The assumption on $v_{1}$ ensures that the right-hand side converges to

$$
\sum_{\substack{I \subset\{1, \ldots, p\} \\|I| \geq 2}} \lambda_{p,|I|} \int \rho\left(\mathrm{d} x_{1}\right) \cdots \rho\left(\mathrm{d} x_{p}\right)\left[f\left(x_{1}^{I}, \ldots, x_{p}^{I}\right)-f\left(x_{1}, \ldots, x_{p}\right)\right] .
$$

It remains to study the 'immigration' part, that is, to establish

$$
\begin{aligned}
\int v_{0}^{n}(\mathrm{~d} y)\left[G\left((1-y) \rho+y \delta_{0}\right)-G(\rho)\right] \\
\quad=\sum_{\substack{J \subset\{1, \ldots, p\} \\
|J| \geq 1}} r_{p,|J|}^{n} \int \rho\left(\mathrm{d} x_{1}\right) \cdots \rho\left(\mathrm{d} x_{p}\right)\left[f\left(x_{1}^{0}, \ldots, x_{p}^{0}\right)-f\left(x_{1}, \ldots, x_{p}\right)\right]
\end{aligned}
$$

with $\left(x_{1}^{0}, \ldots, x_{p}^{0}\right)=\left(z_{1}, \ldots, z_{p}\right)$, where, for all $i \in J, z_{i}=0$ and the values $z_{i}, i \notin J$, listed in the order of $\{1, \ldots, p\} \backslash J$ are the numbers $x_{1}, \ldots, x_{\text {inf } J-1}, x_{\text {inf } J+1}, \ldots, x_{p-|J|}$.

An easy calculation gives

$$
\begin{aligned}
G\left((1-y) \rho+y \delta_{0}\right) & =\prod_{i=1}^{p}\left[(1-y)\left\langle\rho, \phi_{i}\right\rangle+y \phi_{i}(0)\right] \\
& =\sum_{J \subset\{1, \ldots, p\}}(1-y)^{p-|J|} y^{|J|} \prod_{j \notin J}\left\langle\rho, \phi_{j}\right\rangle \prod_{j \in J} \phi_{j}(0)
\end{aligned}
$$

and then, from the identity

$$
\prod_{j \notin J}\left\langle\rho, \phi_{j}\right\rangle \prod_{j \in J} \phi_{j}(0)=\int_{[0,1]^{p}} f\left(x_{1}^{0}, \ldots, x_{p}^{0}\right) \rho\left(\mathrm{d} x_{1}\right) \cdots \rho\left(\mathrm{d} x_{p}\right),
$$

it follows that

$$
\int v_{0}^{n}(\mathrm{~d} y) G\left((1-y) \rho+y \delta_{0}\right)=\sum_{\substack{J \subset\{1, \ldots, p\} \\|J| \geq 1}} r_{p,|J|}^{n} \int \rho\left(\mathrm{d} x_{1}\right) \cdots \rho\left(\mathrm{d} x_{p}\right) f\left(x_{1}^{0}, \ldots, x_{p}^{0}\right) .
$$

Moreover, by passing to the limit in $n \rightarrow \infty$, the right-hand side converges to

$$
\sum_{\substack{J \subset\{1, \ldots, p\} \\|J| \geq 1}} r_{p,|J|} \int \rho\left(\mathrm{d} x_{1}\right) \cdots \rho\left(\mathrm{d} x_{p}\right) f\left(x_{1}^{0}, \ldots, x_{p}^{0}\right) .
$$


It follows that, for $f\left(x_{1}, \ldots, x_{p}\right)=\prod_{i}^{p} \phi_{i}\left(x_{i}\right)$, the process

$$
M_{f}(t):=G_{f}\left(Z_{t}^{0}\right)-\int_{0}^{t} L G_{f}\left(Z_{s}^{0}\right) \mathrm{d} s
$$

is a martingale, where $L$ is the operator defined by

$$
\begin{aligned}
L G_{f}(\rho)= & \sum_{\substack{I \subset\{1, \ldots, p\} \\
|I| \geq 2}} \lambda_{p,|I|} \int \rho\left(\mathrm{d} x_{1}\right) \cdots \rho\left(\mathrm{d} x_{p}\right)\left[f\left(x_{1}^{I}, \ldots, x_{p}^{I}\right)-f\left(x_{1}, \ldots, x_{p}\right)\right] \\
& +\sum_{\substack{J \subset\{1, \ldots, p\} \\
|J| \geq 1}} r_{p,|J|} \int \rho\left(\mathrm{d} x_{1}\right) \cdots \rho\left(\mathrm{d} x_{p}\right)\left[f\left(x_{1}^{0}, \ldots, x_{p}^{0}\right)-f\left(x_{1}, \ldots, x_{p}\right)\right] .
\end{aligned}
$$

Since any continuous function on $[0,1]^{p}$ is the uniform limit of linear combinations of functions of the previous type, we easily conclude that $M_{f}$ is a martingale for any continuous function on $[0,1]^{p}$. The statement claims that, when $c_{0}=c_{1}=0$, the generator has the same integral form as that for finite measures. We assume now that $c_{0}$ and $c_{1}$ are 0 . Let $A_{1}, \ldots, A_{p}$ be i.i.d. variables distributed as $\rho$, and let $x, y \in[0,1]$. Let $\left(\beta_{j}\right)$ and $\left(\beta_{j}^{\prime}\right)$ be two sequences of Bernoulli variables of parameters $x$ and $y$. We set $I:=\left\{j, \beta_{j}=1\right\}$ and $J:=\left\{j, \beta_{j}^{\prime}=1\right\}$. Let $f$ be a continuous function on $[0,1]^{p}$. For $G_{f}(\rho)=\left\langle\rho^{\otimes p}, f\right\rangle$, it is readily checked (see [8]) that

$$
\begin{aligned}
& \int \rho(\mathrm{d} a)\left[G_{f}\left((1-x) \rho+x \delta_{a}\right)-G_{f}(\rho)\right] \\
& =\mathrm{E}\left[f\left(A_{1}^{J}, \ldots, A_{p}^{J}\right)\right]-\mathrm{E}\left[f\left(A_{1}, \ldots, A_{p}\right)\right] \\
& =\sum_{\substack{I \subset\{1, \ldots, p\} \\
|I| \geq 2}} x^{|I|}(1-x)^{p-|I|} \int \rho\left(\mathrm{d} x_{1}\right) \cdots \rho\left(\mathrm{d} x_{p}\right)\left(f\left(x_{1}^{I}, \ldots, x_{p}^{I}\right)-f\left(x_{1}, \ldots, x_{p}\right)\right), \\
& G_{f}\left((1-y) \rho+y \delta_{0}\right)-G_{f}(\rho) \\
& =\mathrm{E}\left[f\left(A_{1}^{0}, \ldots, A_{p}^{0}\right)\right]-\mathrm{E}\left[f\left(A_{1}, \ldots, A_{p}\right)\right] \\
& =\sum_{\substack{J \subset\{1, \ldots, p\} \\
|J| \geq 1}} y^{|J|}(1-y)^{p-|J|} \int \rho\left(\mathrm{d} x_{1}\right) \cdots \rho\left(\mathrm{d} x_{p}\right)\left(f\left(x_{1}^{0}, \ldots, x_{p}^{0}\right)-f\left(x_{1}, \ldots, x_{p}\right)\right) \text {. }
\end{aligned}
$$

We deduce that the process $\left(Z_{t}^{0}\right)$ solves the following martingale problem: for any continuous function $f$ on $[0,1]^{p}, G_{f}\left(Z_{t}^{0}\right)-\int_{0}^{t} \mathrm{~d} s \mathcal{L} G_{f}\left(Z_{s}^{0}\right)$ is a martingale.

Proposition 5.2. The law of the process $\left(Z_{t}^{0}, t \geq 0\right)$ is characterized by the martingale problem of Lemma 5.2, and the operator $\mathcal{L}$ is an extended generator of the process $\left(Z_{t}^{0}, t \geq 0\right)$.

Proof. We will use the same duality argument as in Bertoin and Le Gall [5]. With their notation, we define a class of functions from $\mathcal{M}_{1} \times \mathcal{P}_{p}^{0}$ to $\mathbb{R}$ by

$$
\Phi_{f}:(m, \pi) \in \mathcal{M}_{1} \times \mathcal{P}_{p}^{0} \mapsto \int \delta_{0}\left(\mathrm{~d} x_{0}\right) m\left(\mathrm{~d} x_{1}\right) \cdots m\left(\mathrm{~d} x_{\# \pi-1}\right) f\left(Y\left(\pi ; x_{1}, \ldots, x_{\# \pi-1}\right)\right),
$$

where $f$ is a continuous function on $[0,1]^{p}$ and $Y\left(\pi ; x_{1}, \ldots, x_{\# \pi-1}\right)=\left(y_{1}, \ldots, y_{p}\right)$ is such that $y_{j}=x_{i}$ if $j \in \pi_{i}$ for any $i \geq 0$. 
For a fixed partition $\pi$ in $\mathcal{P}_{n}^{0}$, there exists a function $g$ continuous on $[0,1]^{\# \pi-1}$ with $\mu \mapsto \Phi_{f}(\mu, \pi)=G_{g}(\mu)$, and so $\mathcal{L} \Phi_{f}(\mu, \pi)=\mathcal{L} G_{g}(\mu)$ is well defined. We stress that, for a fixed measure $\mu, \Phi_{f}(\mu, \cdot)$ is a function on $\mathcal{P}_{p}^{0}$. We show the following duality result:

$$
\mathrm{E}\left[\Phi_{f}\left(Z_{0}^{0}, \Pi^{0}(t)\right)\right]=\mathrm{E}\left[\Phi_{f}\left(Z_{t}^{0}, \Pi_{0}^{0}\right)\right] .
$$

By the cocycle property of the stochastic flow involved, it suffices to focus on the process beginning at $Z_{0}^{0}=\lambda$ :

$$
\begin{aligned}
\mathrm{E}_{\lambda}\left[\phi_{f}\left(Z_{t}^{0}, 0_{[p]}\right)\right] & =\mathrm{E}\left[\int_{[0,1]^{p+1}} \delta_{0}\left(\mathrm{~d} x_{0}\right) \mathrm{d} \hat{B}_{0, t}\left(x_{1}\right) \cdots \mathrm{d} \hat{B}_{0, t}\left(x_{p}\right) f\left(x_{1}, \ldots, x_{p}\right)\right] \\
& =\mathrm{E}\left[\int_{[0,1]^{p+1}} \delta_{0}\left(\mathrm{~d} x_{0}\right) \mathrm{d} x_{1} \cdots \mathrm{d} x_{p} f\left(\hat{B}_{0, t}^{-1}\left(x_{1}\right), \ldots, \hat{B}_{0, t}^{-1}\left(x_{p}\right)\right)\right] \\
& =\mathrm{E}\left[f\left(\hat{B}_{0, t}^{-1}\left(V_{1}\right), \ldots, \hat{B}_{0, t}^{-1}\left(V_{p}\right)\right)\right] .
\end{aligned}
$$

Here $\left(V_{i}, 1 \leq i \leq p\right)$ are independent and uniformly distributed on [0,1]. We define, for $1 \leq i \leq \# \Pi_{\mid[p]}^{0}(t)-1$,

$$
V_{i}^{\prime}:=\hat{B}_{0, t}^{-1}\left(V_{j}\right)
$$

for $j \in \Pi_{i \mid[p]}^{0}(t)$. By Lemma 5.1, $\left(V_{i}^{\prime}\right)_{1 \leq i \leq \# \Pi_{\mid[p]}^{0}(t)-1}$ are uniform i.i.d., independent of $\Pi^{0}(t)$, where $\Pi^{0}$ is an $M$-coalescent with rates $\left(x v_{0}(\mathrm{~d} x), x^{2} v_{1}(\mathrm{~d} x)\right)$.

We obtain

$$
\mathrm{E}_{\lambda}\left[\phi_{f}\left(Z_{t}^{0}, 0_{[p]}\right)\right]=\mathrm{E}\left[\int_{[0,1]^{p+1}} \delta_{0}\left(\mathrm{~d} x_{0}\right) \mathrm{d} x_{1} \cdots \mathrm{d} x_{\# \Pi_{\mid[p]}^{0}(t)-1} f\left(y_{1}, \ldots, y_{\# \Pi_{\mid[p]}^{0}(t)-1}\right)\right]
$$

with $y_{j}=x_{i}$ if $j \in \Pi_{i \mid[p]}^{0}(t)$. Thus, we deduce the duality result

$$
\mathrm{E}_{\lambda}\left[\Phi_{f}\left(Z_{t}^{0}, 0_{[p]}\right)\right]=\mathrm{E}\left[\Phi_{f}\left(\lambda, \Pi_{\mid[p]}^{0}(t)\right)\right],
$$

and so $\mathcal{L} \Phi_{f}(\mu, \pi)=\mathcal{L}^{*} \Phi_{f}(\mu, \pi)$.

From Theorem 4.4.2 of [14], this implies uniqueness for the martingale problem, as well as the strong Markov property for the solution.

Remark 5.2. In the case of a standard $M$-coalescent, $Z_{0}^{0}$ is the Lebesgue measure $\lambda$, and we have

$$
Z_{t}^{0}(\mathrm{~d} r)=\left|\Pi_{0}^{0}(t)\right| \delta_{0}(\mathrm{~d} r)+\sum_{i \geq 1}\left|\Pi^{0}(t)\right|_{i}^{\downarrow} \delta_{W_{i}}(\mathrm{~d} r)+\left(1-\sum_{i \geq 0}\left|\Pi^{0}(t)\right|_{i}^{\downarrow}\right) \mathrm{d} r
$$

where $\left(W_{i}, i \geq 1\right)$ are independent uniform and independent of $\Pi^{0}(t)$.

The extinction of the initial types corresponds to the absorption of the GFVI process $\left(Z_{t}^{0}, t \geq 0\right)$ at $\delta_{0}$. Plainly, this event occurs if and only if the measure $\Lambda_{0}$ is not the zero measure and the $M$-coalescent embedded is coming down from infinity. By Theorem 4.1 we know that the coming down from infinity depends only on the measure $\Lambda_{1}$. In terms of the population model, the immigration mechanism, encoded by $\Lambda_{0}:=c_{0} \delta_{0}+x v_{0}(\mathrm{~d} x)$, has no impact on the extinction occurrence, provided of course that $\Lambda_{0}$ is not the zero measure. 


\section{Proof of Theorem 4.1}

Recall the statement of Theorem 4.1. We give a proof based on martingale arguments.

For the $\Lambda$-coalescents (in our setting this corresponds to having $\Lambda_{0} \equiv 0$ ), Schweinsberg studied the mean time of 'coming down from infinity' and concluded using the Kochen-Stone lemma. The proof we give here is based on martingale arguments. To show that the convergence of the series is sufficient for the coming down from infinity, we need to prove Lemma 6.1, which is similar to Proposition 4.9 of [4, p. 202]. The necessary part of the proof does not follow Schweinsberg's ideas. Assuming that the coalescent comes down from infinity and the sum is infinite, we will define a supermartingale (thanks to Lemmas 6.2, 6.3, and 6.4) and find a contradiction (Lemma 6.5).

Lemma 6.1. Let $\left(\Pi^{0}(t), t \geq 0\right)$ be a $\left(\Lambda_{0}, \Lambda_{1}\right)$-coalescent, where $\Lambda_{0}+\Lambda_{1}$ has no mass at 1 . Let us define the fixation time

$$
\zeta:=\inf \left\{t \geq 0, \Pi^{0}(t)=\left\{\mathbb{Z}_{+}, \varnothing, \ldots\right\}\right\}
$$

Define

$$
\phi(n)=\sum_{k=2}^{n}(k-1) C_{n}^{k} \lambda_{n, k}+\Lambda_{0}([0,1]) n .
$$

Then the expectation of the fixation time is bounded by

$$
\mathrm{E}[\zeta] \leq \sum_{n=1}^{\infty} \frac{1}{\phi(n)}
$$

As a consequence, if the series on the right-hand side converges, the fixation time is finite with probability 1 .

Proof. We will study the process of blocks which do not contain 0 : for all $t>0, \Pi^{*}(t):=$ $\left\{\Pi_{1}^{0}(t), \ldots\right\}$. This process is not partition valued. The jump rates of $\# \Pi_{\mid[n]}^{*}(t)$ are easily computed: for $2 \leq k \leq l+1, \# \Pi_{\mid[n]}^{*}$ jumps from $l$ to $l-k+1$ with rate

$$
C_{l}^{k} \lambda_{l, k} \mathbf{1}_{\{k \leq l\}}+C_{l}^{k-1} r_{l, k-1} .
$$

The first term represents the coagulation of $k$ blocks, none of which contain 0 , and the second term represents the disappearance of $k-1$ blocks (coagulation with $\Pi_{0}^{0}$ ). We obtain the infinitesimal generator of $\# \Pi_{\mid[n]}^{*}$ :

$$
G^{[n]} f(l)=\sum_{k=2}^{l} C_{l}^{k} \lambda_{l, k}[f(l-k+1)-f(l)]+\sum_{k=2}^{l+1} C_{l}^{k-1} r_{l, k-1}[f(l-k+1)-f(l)] .
$$

We define

$$
\phi_{1}(n)=\sum_{k=2}^{n}(k-1) C_{n}^{k} \lambda_{n, k} \quad \text { and } \quad \phi_{2}(n)=\sum_{k=2}^{n+1}(k-1) C_{n}^{k-1} r_{n, k-1} .
$$

Using the binomial formula, we obtain $\phi_{2}(n)=\Lambda_{0}([0,1]) n$. We remark that $\phi_{1}$ is an increasing function. Setting $\phi(n)=\phi_{1}(n)+\phi_{2}(n)$, and assuming the convergence of the sum $\sum_{n=1}^{\infty} 1 / \phi(n)$, we define

$$
f(l)=\sum_{k=l+1}^{\infty} \frac{1}{\phi(k)}
$$


The map $\phi$ is increasing; thus, we have $f(l-k+1)-f(l) \geq(k-1) / \phi(l)$, and so

$$
G^{[n]} f(l) \geq \sum_{k=2}^{l+1}\left(C_{l}^{k} \lambda_{l, k}+C_{l}^{k-1} r_{l, k-1}\right) \frac{k-1}{\phi(l)}=1 .
$$

The process $f\left(\# \Pi_{\mid[n]}^{*}(t)\right)-\int_{0}^{t} G^{[n]} f\left(\# \Pi_{\mid[n]}^{*}(s)\right) \mathrm{d} s$ is a martingale. The quantity

$$
\zeta_{n}:=\inf \left\{t ; \# \Pi_{[n]}^{*}(t)=0\right\}
$$

is a finite stopping time. Let $k \geq 1$. Applying the optional stopping theorem to the bounded stopping time $\zeta_{n} \wedge k$, we obtain

$$
\mathrm{E}\left[f\left(\# \Pi_{\mid[n]}^{*}\left(\zeta_{n} \wedge k\right)\right)\right]-\mathrm{E}\left[\int_{0}^{\zeta_{n} \wedge k} G^{[n]} f\left(\# \Pi_{\mid[n]}^{*}(s)\right) \mathrm{d} s\right]=f(n) .
$$

With the inequality $G^{[n]} f(l) \geq 1$, we deduce that

$$
\mathrm{E}\left[\zeta_{n} \wedge k\right] \leq \mathrm{E}\left[f\left(\# \Pi_{\mid[n]}^{*}\left(\zeta_{n} \wedge k\right)\right)\right]-f(n) .
$$

By monotone convergence and Lebesgue's theorem, we have $\mathrm{E}\left[\zeta_{n}\right] \leq f(0)-f(n)$. Passing to the limit in $n$, we have $\zeta_{n} \uparrow \zeta_{\infty}:=\inf \{t ; \# \Pi(t)=1\}$ and $f(n) \rightarrow 0$; thus,

$$
\mathrm{E}\left[\zeta_{\infty}\right] \leq f(0)=\sum_{k=1}^{\infty} \frac{1}{\phi(k)}
$$

By simple series comparisons, we deduce the sufficient part of the theorem. Plainly, $\phi(n) \geq$ $\phi_{1}(n)$, and if the series $\sum_{n=1}^{\infty} 1 / \phi_{1}(n)$ converges then, by Lemma 6.1, the $M$-coalescent comes down from infinity.

To show that the convergence of the series is necessary for the coming down, we must look more precisely at the behaviour of the jumps. The following technical lemmas show that, when a distinguished coalescent comes down from infinity, there is a finite number of jumps which decrease by half or more the number of blocks. Lemma 6.3 will allow us to study the process of the number of blocks before the first of these times. Assuming that the sum $\sum_{n=2}^{\infty} 1 / \phi(n)=\infty$ is infinite, we will define a supermartingale in Lemma 17 and find a contradiction by applying the optional stopping theorem.

We have already seen that, as for the $\Lambda$-coalescent, a way to understand the dynamics of a $\left(\Lambda_{0}, \Lambda_{1}\right)$-coalescent, when $\Lambda_{0}$ and $\Lambda_{1}$ have no mass at 0 , is to imagine drawing an infinite sequence of Bernoulli variables at each jump time, with parameter $x$ controlled by the measures $v_{0}(\mathrm{~d} x)=x^{-1} \Lambda_{0}(\mathrm{~d} x)$ and $\nu_{1}(\mathrm{~d} x)=x^{-2} \Lambda_{1}(\mathrm{~d} x)$. The following technical lemma allows us to estimate the chance for a Bernoulli vector to have more than half of its terms equal to 1 .

Lemma 6.2. Let $\left(X_{1}, X_{2}, \ldots\right)$ be independent Bernoulli variables with parameter $x \in\left[0, \frac{1}{4}\right)$. Defining $S_{n}^{(x)}=X_{1}+\cdots+X_{n}$, for every $n_{0}$, there is the bound

$$
\mathrm{P}\left[\text { there exists } n \geq n_{0}, S_{n}^{(x)}>\frac{n}{2}\right] \leq \frac{\exp \left(-n_{0} f(x)\right)}{1-\exp (-f(x))}
$$

with $f(x) \sim \frac{1}{2} \log (1 / x)$ when $x \rightarrow 0$. 
Proof. By Markov's inequality, for all $t>0$,

$$
\mathrm{P}\left[S_{n}^{(x)} \geq \frac{n}{2}\right] \leq \mathrm{e}^{-n t / 2} \mathrm{E}\left[\mathrm{e}^{t S_{n}^{(x)}}\right]=\exp \left(-n\left[\frac{t}{2}-\log \left(x \mathrm{e}^{t}+1-x\right)\right]\right) .
$$

Applying this inequality for $t=\log (1 / x)$, we obtain $\mathrm{P}\left[S_{n}^{(x)} \geq n / 2\right] \leq \mathrm{e}^{-n f(x)}$, where

$$
f(x)=\frac{1}{2} \log \left(\frac{1}{x}\right)-\log (2-x) .
$$

The function $f$ is nonnegative on $\left(0, \frac{1}{4}\right)$, and so we obtain the convergence of the geometric sum

$$
\mathrm{P}\left[\text { there exists } n \geq n_{0}, S_{n}^{(x)}>\frac{n}{2}\right] \leq \sum_{n \geq n_{0}} \exp (-n f(x))=\frac{\exp \left(-n_{0} f(x)\right)}{1-\exp (-f(x))} .
$$

Moreover, we have $f(x) \sim \frac{1}{2} \log (1 / x)$ as $x \rightarrow 0^{+}$.

Lemma 6.3. Assume that the $M$-coalescent comes down from infinity. With probability 1 , we have

$$
\tau:=\inf \left\{t>0, \# \Pi^{0}(t)<\frac{\# \Pi^{0}(t-)}{2}\right\}>0 .
$$

Moreover, if we define $\tau_{n}:=\inf \left\{t>0, \# \Pi_{\mid[n]}^{0}(t)<\# \Pi_{\mid[n]}^{0}(t-) / 2\right\}$ then the sequence of stopping times $\tau_{n}$ converges to $\tau$ almost surely.

Proof. Obviously, binary coagulations play no role in the statement and we may assume that $\Lambda_{0}(\{0\})=0$ and $\Lambda_{1}(\{0\})=0$. Let $N$ be a Poisson measure with intensity $\mathrm{d} t \otimes\left(x^{-1} \Lambda_{0}(\mathrm{~d} x)+\right.$ $\left.x^{-2} \Lambda_{1}(\mathrm{~d} x)\right)$. Recall the notation in Lemma 6.2. Let $n_{0} \geq 4$. We will show that

$$
N\left(\left\{(t, x) ; t \leq 1 ; \text { there exists } n \geq n_{0}, S_{n}^{(x)} \geq \frac{n}{2}\right\}\right)<\infty .
$$

By Proposition 4.1, \# $\Pi^{0}(\varepsilon)<\infty$ almost surely for every $\varepsilon>0$. We will then deduce that there is a finite number of jump times before 1 where more than half of the blocks coagulate. By the Feller property and, therefore, the regularity of the paths of $\left(\Pi^{0}(t)\right)_{t \geq 0}, \Pi^{0}(0+)=\Pi^{0}(0)$ and 0 is not a jump time; then, almost surely, $\tau>0$. Moreover, $\# \Pi^{0}(\tau-)<\infty$ and so, for all $n \geq \# \Pi^{0}(\tau-), \tau_{n}=\tau$. We deduce that $\tau_{n} \rightarrow \tau$ as $n \rightarrow \infty$ almost surely.

By Poissonian calculations, we obtain

$$
\begin{aligned}
& \mathrm{E}\left[N\left(\left\{(t, x) ; t \leq 1 ; \text { there exists } n \geq n_{0}, S_{n}^{(x)}>\frac{n}{2}\right\}\right)\right] \\
& \quad=\int_{0}^{1}\left(v_{0}+v_{1}\right)(\mathrm{d} x) \mathrm{P}\left[\text { there exists } n \geq n_{0}, S_{n}^{(x)}>\frac{n}{2}\right] .
\end{aligned}
$$

By Lemma 6.2 we obtain

$$
\begin{aligned}
& \int_{0}^{1}\left(v_{0}+v_{1}\right)(\mathrm{d} x) \mathrm{P}\left[\text { there exists } n \geq n_{0}, S_{n}^{(x)}>\frac{n}{2}\right] \\
& \leq \int_{0}^{1 / 4}\left(v_{0}+v_{1}\right)(\mathrm{d} x) \frac{\exp \left(-n_{0} f(x)\right)}{1-\exp (-f(x))}+\int_{1 / 4}^{1}\left(v_{0}+v_{1}\right)(\mathrm{d} x) .
\end{aligned}
$$


On the one hand,

$$
\int_{0}^{1 / 4}\left(v_{0}+v_{1}\right)(\mathrm{d} x) \frac{\exp \left(-n_{0} f(x)\right)}{1-\exp (-f(x))}<\infty
$$

because the integrand is bounded by $8 x^{n_{0} / 2}$ and $n_{0} \geq 4$. On the other hand,

$$
\int_{1 / 4}^{1}\left(v_{0}+v_{1}\right)(\mathrm{d} x) \leq \int_{1 / 4}^{1} x^{-2} x^{2}\left(v_{0}+v_{1}\right)(\mathrm{d} x) \leq 16 \int_{0}^{1} x^{2}\left(v_{0}+v_{1}\right)(\mathrm{d} x)<\infty .
$$

This completes the proof.

Assuming that the coalescent comes down from infinity and that $\sum_{n \geq 1} 1 / \phi(n)=\infty$, we can define a supermartingale. We will find a contradiction using the optional stopping theorem.

We define the decreasing function

$$
f(n)=\exp \left(-\sum_{k=1}^{n+1} \frac{1}{\phi(k)}\right)
$$

where $\phi(n)=\phi_{1}(n)+\phi_{2}(n)$, with $\phi_{1}(n)$ and $\phi_{2}(n)$ defined as in Lemma 6.1.

Lemma 6.4. There exists a constant $C>0$ such that, for all $n \geq 1,\left(\mathrm{e}^{-C t} f\left(\# \Pi_{\mid[n]}^{*}(t)\right)\right)_{t \leq \tau_{n}}$ is a nonnegative supermartingale.

Proof. We recall that the generator of $\left(\# \Pi_{\mid[n]}^{*}(t)\right)_{t \geq 0}$ is

$$
G^{[n]} g(l)=\sum_{k=2}^{l+1}\left[C_{l}^{k} \mathbf{1}_{\{k \leq l\}} \lambda_{l, k}+C_{l}^{k-1} r_{l, k-1}\right][g(l-k+1)-g(l)] .
$$

Stopping the process at $\tau_{n}$, the jump times where more than half of the blocks coagulate are ignored, and the generator of the stopped process is

$$
A^{[n]} g(l)=\sum_{k=2}^{l / 2+1}\left[C_{l}^{k} \mathbf{1}_{\{k \leq l / 2\}} \lambda_{l, k}+C_{l}^{k-1} r_{l, k-1}\right][g(l-k+1)-g(l)] .
$$

We set $\Psi(n)=\int_{0}^{1}\left(\mathrm{e}^{-n x}-1+n x\right) v_{1}(\mathrm{~d} x)$. An easy verification allows us to claim the existence of a $c>0$ such that $c \Psi(q) \leq \phi_{1}(q) \leq \Psi(q)$ (see the remark on page 170 of [7]); moreover,

$$
\frac{\Psi(q)}{q}=\int_{0}^{1}\left(1-\mathrm{e}^{-q x}\right) v_{1}((x, 1)) \mathrm{d} x \rightarrow \int_{0}^{1} x v_{1}(\mathrm{~d} x)>0 \quad \text { as } q \rightarrow \infty .
$$

Plainly, $h(q)=\Psi(q) / q$ is a concave function, so $h(q / 2) \geq h(q) / 2$ and $\Psi(q / 2) \geq \Psi(q) / 4$.

Let us compute

$$
A^{[n]} f(l)=\sum_{k=2}^{l / 2+1}\left[C_{l}^{k} \mathbf{1}_{\{k \leq l / 2\}} \lambda_{l, k}+C_{l}^{k-1} r_{l, k-1}\right] f(l)\left[\exp \left(\sum_{l-k+2}^{l} \frac{1}{\phi(j)}\right)-1\right] .
$$

We have

$$
\sum_{l-k+2}^{l} \frac{1}{\phi(j)} \leq \frac{k-1}{\phi(l-k+2)} \leq \frac{k-1}{\phi(l / 2)} \quad \text { for all } k \leq \frac{l}{2}+1
$$


and $\mathrm{e}^{x}-1 \leq c x$ for small $x$. Then, for large $l$,

$$
A^{[n]} f(l) \leq c f(l) \sum_{k=1}^{l / 2+1}\left[C_{l}^{k} \lambda_{l, k}+C_{l}^{k-1} r_{l, k-1}\right] \frac{k-1}{\phi(l / 2)} .
$$

Thus, $A^{[n]} f(l) \leq c f(l) \phi(l) / \phi(l / 2)$.

By definition, $\phi(l)=\phi_{1}(l)+\Lambda_{0}([0,1]) l$. Moreover, $l / \phi_{1}(l)$ is bounded (it converges to $\left.\left(\int_{[0,1]} x v_{1}(\mathrm{~d} x)\right)^{-1}\right)$ and, from the inequalities

$$
\frac{c \Psi(l)}{l} \leq \frac{\phi_{1}(l)}{l} \leq \frac{\Psi(l)}{l} \text { and } \Psi\left(\frac{l}{2}\right) \geq \frac{\Psi(l)}{4},
$$

we deduce that, for some constant $C>0$,

$$
\begin{aligned}
\frac{\phi(l)}{\phi(l / 2)} & \leq \frac{\phi_{1}(l)+\Lambda([0,1]) l}{\phi_{1}(l / 2)} \\
& =\frac{\phi_{1}(l)}{\phi_{1}(l / 2)}+\left(\Lambda_{0}([0,1])\right) \frac{l}{\phi_{1}(l)} \frac{\phi_{1}(l)}{\phi_{1}(l / 2)} \\
& \leq \frac{4}{c}\left[1+\Lambda_{0}([0,1]) \frac{l}{\phi_{1}(l)}\right] \\
& \leq C .
\end{aligned}
$$

Therefore, $A^{[n]} f(l) \leq C f(l)$ and $\left(\mathrm{e}^{-C t} f\left(\# \Pi_{\mid[n]}^{*}(t)\right)\right)_{t \leq \tau}$ is a supermartingale.

Lemma 6.5. If $\sum_{n \geq 1} 1 / \phi(n)=\infty$ then $\Pi^{0}$ does not come down from infinity.

Proof. Assume that the $M$-coalescent comes down from infinity. By Proposition 4.1 we know that $T=0$ almost surely. Let $T_{j}^{(n)}:=\inf \left\{t>0 ; \# \Pi_{\mid[n]}^{*}(t) \leq j\right\}$. We apply to the previous supermartingale, the optional stopping theorem at time $T_{j}^{(n)} \wedge \tau_{n}$ and obtain

$$
\mathrm{E}\left[\exp \left(-c T_{j}^{(n)} \wedge \tau_{n}\right) f\left(\# \Pi_{\mid[n]}^{*}\left(\tau_{n} \wedge T_{j}^{(n)}\right)\right)\right] \leq f(n) .
$$

Passing to the limit with $n \uparrow \infty, f(n) \rightarrow 0, T_{j}^{(n)} \uparrow T_{j}$, and $\tau_{n} \rightarrow \tau>0$ (by Lemma 6.3). The time $T_{j}$ is strictly positive for some $j$. Then $\tau \wedge T_{j}>0$, \# $\Pi^{*}\left(\tau \wedge T_{j}\right)<\infty$, and, thus, $f\left(\# \Pi^{*}\left(\tau \wedge T_{j}\right)\right)>0$ almost surely. We have

$$
\mathrm{E}\left[\exp \left(-c T_{j} \wedge \tau\right)\right]=0 .
$$

Then $T_{j}=\infty$ almost surely, which is not possible on $T<\infty$.

It remains to establish that the convergence of the series is necessary for the coming down from infinity. When $\Lambda_{0}(\{0\})=0$, the previous lemma claims that if the $M$-coalescent comes down from infinity then $\sum_{n \geq 1} 1 / \phi(n)<\infty$. It suffices to show that

$$
\sum_{n \geq 2} \frac{1}{\phi_{1}(n)+\Lambda_{0}([0,1]) n}<\infty \Longrightarrow \sum_{n \geq 2} \frac{1}{\phi_{1}(n)}<\infty .
$$

The sequence $(\Psi(n) / n)_{n \geq 1}$ is increasing and tends to $\int_{0}^{1} x^{-1} \Lambda_{1}(\mathrm{~d} x)$ (possibly infinite). From the inequality $c \Psi(n) / n \leq \phi_{1}(n) / n \leq \Psi(n) / n$, we find that $n / \phi_{1}(n)$ is bounded. It follows that

$$
\frac{1}{\phi_{1}(n)+\Lambda_{0}([0,1]) n}=\frac{1}{\phi_{1}(n)\left(1+\Lambda_{0}([0,1]) n / \phi_{1}(n)\right)} \geq c \frac{1}{\phi_{1}(n)}
$$

for some constant $c>0$.

We then get the necessary part, and combining the results, Theorem 4.1 is deduced. 


\section{Acknowledgements}

This work forms part of my PhD thesis. I would like to thank my advisor Jean Bertoin for introducing this subject, his useful advice, and his encouragement. I also thank the anonymous referee for his/her very careful reading and helpful suggestions.

\section{References}

[1] Aldous, D. J. (1985). Exchangeability and related topics. In École d'été de Probabilités de Saint-Flour, XIII1983 (Lecture Notes Math. 1117), Springer, Berlin, pp. 1-198.

[2] Berestycki, J., Berestycki, N. AND Limic, V. (2011). Asymptotic sampling formulae and particle system representations for $\Lambda$-coalescents. Submitted.

[3] BerESTYCKI, N. (2010). Recent progress in coalescent theory. Math. Surveys 16, 193pp.

[4] Bertoin, J. (2006). Random Fragmentation and Coagulation Processes (Camb. Stud. Adv. Math. 102), Cambridge University Press.

[5] Bertoin, J. And Le Gall, J.-F. (2003). Stochastic flows associated to coalescent processes. Prob. Theory Relat. Fields 126, 261-288.

[7] Bertoin, J. and Le Gall, J.-F. (2006). Stochastic flows associated to coalescent processes. III. Limit theorems. Illinois J. Math. 50, 147-181.

[8] Birkner, M. et al. (2005). Alpha-stable branching and beta-coalescents. Electron. J. Prob. 10, 303-325.

[12] Donnelly, P. AND Joyce, P. (1991). Consistent ordered sampling distributions: characterization and convergence. Adv. Appl. Prob. 23, 229-258.

[13] Donnelly, P. And Kurtz, T. G. (1999). Particle representations for measure-valued population models. Ann. Prob. 27, 166-205.

[14] Ethier, S. N. And Kurtz, T. G. (1986). Markov Processes. John Wiley, New York.

[15] Gnedin, A. V. (1997). The representation of composition structures. Ann. Prob. 25, 1437-1450.

[16] KawazU, K. and Watanabe, S. (1971). Branching processes with immigration and related limit theorems. Theory Prob. Appl. 16, 36-54.

[21] Pitman, J. (1995). Exchangeable and partially exchangeable random partitions. Prob. Theory Relat. Fields 102, 145-158.

[22] Pitman, J. (1999). Coalescents with multiple collisions. Ann. Prob. 27, 1870-1902.

[23] Pitman, J. (2006). Combinatorial Stochastic Processes (Lecture Notes Math. 1875), Springer, Berlin.

[24] Sagitov, S. (1999). The general coalescent with asynchronous mergers of ancestral lines. J. Appl. Prob. 36, 1116-1125.

[25] Schweinsberg, J. (2000). A necessary and sufficient condition for the $\Lambda$-coalescent to come down from infinity. Electron. Commun. Prob. 5, 1-11.

[26] Schweinsberg, J. (2000). Coalescents with simultaneous multiple collisions. Electron. J. Prob. 5, 50pp. 